\title{
RATE OF CONVERGENCE OF A STOCHASTIC PARTICLE METHOD FOR THE KOLMOGOROV EQUATION WITH VARIABLE COEFFICIENTS
}

\author{
PIERRE BERNARD, DENIS TALAY, AND LUCIANO TUBARO
}

\begin{abstract}
In a recent paper, E. G. Puckett proposed a stochastic particle method for the nonlinear diffusion-reaction PDE in $[0, T] \times \mathbb{R}$ (the so-called
\end{abstract} "KPP" (Kolmogorov-Petrovskii-Piskunov) equation):

$$
\left\{\begin{array}{l}
\frac{\partial u}{\partial t}=A u=\Delta u+f(u), \\
u(0, \cdot)=u_{0}(\cdot),
\end{array}\right.
$$

where $1-u_{0}$ is the cumulative function, supposed to be smooth enough, of a probability distribution, and $f$ is a function describing the reaction. His justification of the method and his analysis of the error were based on a splitting of the operator $A$. He proved that, if $h$ is the time discretization step and $N$ the number of particles used in the algorithm, one can obtain an upper bound of the norm of the random error on $u(T, x)$ in $L^{1}(\Omega \times \mathbb{R})$ of order $1 / N^{1 / 4}$, provided $h=\mathscr{O}\left(1 / N^{1 / 4}\right)$, but conjectured, from numerical experiments, that it should be of order $\mathscr{O}(h)+\mathscr{O}(1 / \sqrt{N})$, without any relation between $h$ and $N$.

We prove that conjecture. We also construct a similar stochastic particle method for more general nonlinear diffusion-reaction-convection PDEs

$$
\left\{\begin{array}{l}
\frac{\partial u}{\partial t}=L u+f(u), \\
u(0, \cdot)=u_{0}(\cdot),
\end{array}\right.
$$

where $L$ is a strongly elliptic second-order operator with smooth coefficients, and prove that the preceding rate of convergence still holds when the coefficients of $L$ are constant, and in the other case is $\mathscr{\sigma}(\sqrt{h})+\mathscr{O}(1 / \sqrt{N})$.

The construction of the method and the analysis of the error are based on a stochastic representation formula of the exact solution $u$.

\section{INTRODUCTION}

1.1. Setting of the problem. In a recent paper [12], E. G. Puckett proposed a stochastic particle method for the nonlinear PDE in $[0, T] \times \mathbb{R}$ :

$$
\left\{\begin{array}{l}
\frac{\partial u}{\partial t}=A u=\Delta u+f(u), \\
u(0, \cdot)=u_{0}(\cdot),
\end{array}\right.
$$

where $1-u_{0}$ is the cumulative function, supposed to be smooth enough, of a probability distribution, and $f$ is a function satisfying properties ensuring, in particular, that the solution $u(t, x)$ takes values in $[0,1]$.

Received by the editor February 22, 1993 and, in revised form, September 13, 1993.

1991 Mathematics Subject Classification. Primary 35K57, 60J15, 60J60, 65M15. 
His justification of the method and his analysis of the error were based on a splitting of the operator $A$; a rough presentation of the algorithm is the following:

(A) Initialization: One locates $N$ particles on the real axis at positions $x_{0}^{i}$ with weights $\omega_{0}^{i}(i=1, \ldots, N)$ of order $\frac{1}{N}$, such that the function ${ }^{1} \bar{u}(0, x)=$ $\sum_{i=1}^{N} \omega_{0}^{i} H\left(x_{0}^{i}-x\right)$ is a good approximation of $u_{0}$ in $L^{1}(\mathbb{R})$.

(B) Approximation of the reaction: One numerically solves the ODE

$$
\left\{\begin{array}{l}
\frac{\partial v}{\partial t}=f(v) \\
v(0, \cdot)=\bar{u}(0, \cdot)
\end{array}\right.
$$

on a time interval of length $h$ (this operation changes the weights of the particles).

(C) Approximation of the diffusion: One numerically solves

$$
\left\{\begin{array}{l}
\frac{\partial w}{\partial t}=\Delta w \\
w(0, \cdot)=v(h, \cdot)
\end{array}\right.
$$

by randomly and independently moving the particles, considered as independent Brownian particles, during a time interval equal to $h$, each particle keeping its own weight.

(D) Computation of the approximate solution: The value at time $h$ and point $x$ of the approximate solution, $\bar{u}(h, x)$, is obtained by adding the weights of all the particles which are at the right-hand side of $x$.

(E) Iteration: At each time step, one performs the operations (B) (using $\bar{u}(p h, \cdot)$ instead of $\bar{u}(0, \cdot)),(\mathrm{C})$, and (D).

The upper bound of the random error on $u(T, x)$ in $L^{1}(\Omega \times \mathbb{R})$ is shown to be of order $1 / N^{1 / 4}$, provided $h=\mathscr{O}\left(1 / N^{1 / 4}\right)$.

In the last section of the paper, Puckett presents numerical results which obviously show that this estimation is very pessimistic and conjectures that the rate of convergence should be of order $\mathscr{O}(h)+\mathscr{O}(1 / \sqrt{N})$, without any relation between $h$ and $N$.

We tried to prove this conjecture by keeping the idea of the splitting but changing the technique used by Puckett to obtain some of his estimations. We could obtain a better rate of convergence than $1 / N^{1 / 4}$ (we got $1 / N^{2 / 7}$ provided $h$ is of order $1 / N^{2 / 7}$ ), but we could neither get the right one, nor avoid a relation between $h$ and $N$, mainly because we had to sum up the approximation errors made at each step on the solution of the following PDE, where the initial condition $\bar{u}(p h, \cdot)$ is the approximate solution computed at step $p=1, \ldots, \frac{T}{h}$ :

$$
\left\{\begin{array}{l}
\frac{\partial w}{\partial t}=\Delta w \\
w(0, \cdot)=\bar{u}(p h, \cdot)
\end{array}\right.
$$

and these local errors appear to be of order $\frac{h^{1 / 4}}{\sqrt{N}}$.

Besides, the notion of splitting does not represent the basic operation of the algorithm, which is the approximation of the measure $\frac{\partial}{\partial x} u(t, x) d x$ by a linear combination of Dirac measures at points defined by the current positions of the particles, and coefficients in the combination equal to the respective weights.

\footnotetext{
${ }^{1}$ In the sequel, $H$ will denote the Heaviside function: $H(y)=0$ for $y<0, H(y)=1$ for $y \geq 0$.
} 
Thus, we were led to change our point of view.

Our objective was also to extend the algorithm to more general nonlinear reaction-diffusion-convection PDEs, namely,

$$
\left\{\begin{array}{l}
\frac{\partial u}{\partial t}=A u=L u+f(u), \\
u(0, \cdot)=u_{0}(\cdot),
\end{array}\right.
$$

where $L$ is a strongly elliptic second-order operator with smooth coefficients. A natural question is then: what must be the law of the motion of the particles? A natural attempt is to move the particles according to the law of a diffusion process whose infinitesimal generator is $L$, but then one can see the computed solution (considered as a wave) propagate in the opposite direction to the propagation direction of the exact solution!

The answers to this question and to the determination of the rate of convergence of the algorithm that we will construct (which reduces to the Puckett algorithm when $L=\Delta$ ) are based upon an interpretation of the method completely different from the splitting of $A$ in (1.1), and an analysis of the error completely different from Puckett's one. The main tool will be a probabilistic representation formula of the exact solution, which will be used to get estimates on the rate of convergence. We emphasize that, applied to the Puckett algorithm for the KPP equation, our estimates below prove Puckett's conjecture.

We also stress that the stochastic particle algorithm we analyze is not the only one that can be developed for nonlinear reaction-convection-diffusion equations. In particular, Sherman and Peskin have proposed a numerical method (without proving convergence) in [14], based upon the simulation of branching Brownian motions; the term $f(u)$ is used to describe the law of the branching. For the convergence and the analysis of this algorithm, see the papers of Chauvin and Rouault $[5,4,3]$. The main difference between the two algorithms is the following: the Sherman-Peskin particles have constant weights, but are highly dependent (they are the living particles of the branching process); the Puckett particles are independent, but the weights are dependent. For a finite horizon problem, the Puckett method seems to be simpler to implement and easier to use on a parallel computer; but if the problem is, for example, to study the asymptotic propagation velocity of a wave, then the Puckett algorithm cannot be efficient, because there is no reason at all for it to be stable (see our results on the rate of convergence); in that case, the Sherman-Peskin method must be preferred, since it is naturally related to the evolution of the solution, the particles concentration being large where the gradient of the solution is large.

Our paper is organized as follows: in $\S 2$, we state our hypotheses and we present a collection of elementary results, which are frequently used in the sequel; then, in $\S 3$, we establish an original stochastic representation of the solution of the above nonlinear PDE; this formula permits us to construct a stochastic particle method, which reduces to the splitting method of Puckett when the coefficients of $L$ are constant; in $\S 4$, we state our result on the rate of convergence; before proving it ( $\S \S 6$ and 7$)$, we need to study in a precise way how dependent the weights of the particles are: this is done in $\S 5$. Finally $(\S 8)$, we consider the special case of constant coefficients. The Supplement contains some of the proofs $(\S 10)$ and an Appendix devoted to reaction-diffusion-convection PDEs.

Our numerical experiments for nonconstant-coefficient examples do not add 
information to the excellent last section of the paper of Puckett (devoted to the KPP equation), so that we refer to it.

1.2. Notation, conventions. In the sequel, $C$ will denote any deterministic strictly positive constant independent of the time discretization step $h$ and the number of particles $N$ (but, most often, it will depend on $T$ ).

We will always assume $h \in(0,1)$, of the form $\frac{T}{M}$, where $M$ is an integer.

When a stochastic process $\left(X_{t}\right)$ is such that $X_{0}=y$ a.s. for some real number $y$, we will often write $\left(X_{t}(y)\right)$.

When we write $\mathscr{O}(h)$ or $\mathscr{O}\left(\frac{1}{N}\right)$, etc., it must be understood that the quantity involved (which may be random) can be bounded, uniformly in $\omega$ if it is random, by, respectively, $C h$ or $\frac{C}{N}$, the constant $C$ being deterministic and uniform with respect to $h$ and $N$.

\section{HyPOTHESES AND ELEMENTARY RESULTS}

2.1. Hypotheses. We make the following assumptions:

(H1) $f$ is a $C^{2}$ function on $[0,1]$ such that $f(0)=f(1)=0, f(u) \geq 0$ for $u \in[0,1]$ (therefore, $\frac{f(u)}{u}$ is bounded in $(0,1]$ and continuous in 0$)$;

(H2) $b, \sigma$ are two bounded $C^{\infty}$ functions; any derivative of any order is assumed to be a bounded function; $\sigma$ is bounded below by a strictly positive constant;

(H3) $1-u_{0}$ is the cumulative function of a probability distribution.

In the Appendix (see the Supplement), we recall that, under $(\mathrm{H} 1),(\mathrm{H} 2),(\mathrm{H} 3)$, for any $T>0$, there exists a unique classical solution in $(0, T] \times \mathbb{R}$, taking values in $[0,1]$, to the problem

$$
\left\{\begin{array}{l}
\frac{\partial u}{\partial t}=L u+f(u), \\
\lim _{t \rightarrow 0} u(t, \cdot)=u_{0}(\cdot) \quad \text { at every continuity point of } u_{0},
\end{array}\right.
$$

where

$$
L=b(x) \frac{\partial}{\partial x}+\frac{1}{2} \sigma^{2}(x) \frac{\partial^{2}}{\partial x^{2}} .
$$

In the sequel, we will often need an additional assumption on $u_{0}$ :

(H4) $u_{0}$ is of class $\mathscr{C}_{b}^{\infty}(\mathbb{R})$, and there exist strictly positive constants $C_{1}, C_{2}$ such that, for any $x$ in $\mathbb{R},\left|u_{0}^{\prime}(x)\right| \leq C_{1} e^{-C_{2} x^{2}}$, or

(H5) $u_{0}$ is of the form

$$
u_{0}(x)=\sum_{i=1}^{N} \omega_{0}^{i} H\left(x_{0}^{i}-x\right),
$$

where the $\omega_{0}^{i}$ 's are positive and such that

$$
\sum_{i=1}^{N} \omega_{0}^{i}=1
$$

2.2. Elementary results. In this subsection, we will state easy consequences of quite classical results, needed in the sequel.

We begin with the obvious (but useful) inequality:

$$
\forall x>0, \quad \int_{x}^{+\infty} e^{-y^{2}} d y \leq C e^{-x^{2}} .
$$


We derive some consequences of $(\mathrm{H} 2)$.

Let $\left(\zeta_{t}\right)$ be a diffusion process whose infinitesimal generator has bounded and $C_{b}^{\infty}(\mathbb{R})$ coefficients $b_{0}$ and $\sigma_{0}$, and is strictly elliptic.

We have the well-known property (see, for instance, Friedman [6]): under (H2), there exist $C>0, \lambda>0$ such that, for the density $p_{t}(y, z)$ of the law of $\zeta_{t}(y) \quad(y, z \in \mathbb{R}, 0<t \leq T)$, we have

$$
p_{t}(y, z) \leq \frac{C}{\sqrt{t}} \exp \left(-\frac{(z-y)^{2}}{2 \lambda t}\right) .
$$

Therefore (for (iii) we apply (2.2)):

Corollary 2.1. Let $\left(\zeta_{t}\right)$ be a diffusion process whose infinitesimal generator has bounded and $C_{b}^{\infty}(\mathbb{R})$ coefficients, and is strictly elliptic. Then there exist $C>0$ and $\lambda>0$ such that for all $t$ with $0<t \leq T$ and for all $x, y \in \mathbb{R}$, we have

$$
\mathbb{P}\left(\zeta_{t}(y)<x\right) \leq \frac{C}{\sqrt{t}} \int_{-\infty}^{x} \exp \left(-\frac{(z-y)^{2}}{2 \lambda t}\right) d z
$$

$$
\mathbb{P}\left(\zeta_{t}(y)>x\right) \leq \frac{C}{\sqrt{t}} \int_{x}^{+\infty} \exp \left(-\frac{(z-y)^{2}}{2 \lambda t}\right) d z
$$

$$
\mathbb{P}\left(\zeta_{t}(y)<x\right) \mathbb{P}\left(\zeta_{t}(y)>x\right) \leq C \exp \left(-\frac{(y-x)^{2}}{2 \lambda t}\right) .
$$

Besides, we observe that, under the above hypotheses, there exists a constant $C>0$ such that

$$
\forall 0<t \leq T, \quad \forall y \in \mathbb{R}, \quad \mathbb{E}\left|\xi_{t}(y)-y\right| \leq C \sqrt{t}
$$

and $^{2}$

$$
\forall t>0, \quad \lim _{y \rightarrow+\infty} \zeta_{t}(y)=+\infty \text { a.s. }
$$

because the function $y \rightarrow \xi_{t}(y)$ is a.s. increasing since its derivative is an exponential.

Lemma 2.2. Under the above hypotheses on $\left(\xi_{t}\right)$, there exists $a C>0$ such that for any $T \geq t>0$, the probability density $p_{t}(x, y)$ of the law of $\xi_{t}(x)$ satisfies

$$
\left|\int_{\mathbf{R}} p_{t}(x, y) d x-1\right| \leq C t
$$

for any $y \in \mathbb{R}$.

The proof is in the Supplement.

We recall that we denote by $b_{0}(\cdot)$ and $\sigma_{0}(\cdot)$, respectively, the drift and the diffusion coefficients of $\left(\zeta_{t}\right)$. Let $\left(B_{t}\right)$ be a standard real Brownian motion.

The Euler scheme is defined by

$$
\hat{\zeta}_{p+1}=\hat{\zeta}_{p}+b_{0}\left(\hat{\zeta}_{p}\right) h+\sigma_{0}\left(\hat{\zeta}_{p}\right)\left(B_{(p+1) h}-B_{p h}\right)
$$

\footnotetext{
${ }^{2}$ See Kunita [8, Chapter 2], e.g., for the diffeomorphism property of stochastic flows associated with stochastic differential equations.
} 
and the Milshtein scheme is defined by

$$
\begin{aligned}
\bar{\zeta}_{p+1}= & \bar{\zeta}_{p}+b_{0}\left(\bar{\zeta}_{p}\right) h+\sigma_{0}\left(\bar{\zeta}_{p}\right)\left(B_{(p+1) h}-B_{p h}\right) \\
& +\frac{1}{2} \sigma_{0}\left(\bar{\zeta}_{p}\right) \sigma_{0}^{\prime}\left(\bar{\zeta}_{p}\right)\left(\left(B_{(p+1) h}-B_{p h}\right)^{2}-h\right) .
\end{aligned}
$$

We now recall a result on the convergence rate (the first part is easy to show; the second is due to Milshtein [9]).

Proposition 2.3. Suppose that the functions $b_{0}$ and $\sigma_{0}$ are of class $C^{\infty}$, and that any derivative is uniformly bounded. For the Milshtein scheme, we have

(i) For any $k \in \mathbb{N}^{*}$ and for any initial condition $\zeta_{0}$ such that $\mathbb{E}\left|\zeta_{0}\right|^{2 k}<\infty$, there exists a strictly positive constant $C$ (depending only on $T$, and the bounds of $b_{0}, \sigma_{0}$, and their two first derivatives) such that

$$
\begin{gathered}
\forall M \in \mathbb{N}^{*}, \quad \forall h=\frac{T}{M}, \quad \forall p=1, \ldots, M, \\
E\left|\zeta_{p h}\right|^{2 k}+\mathbb{E}\left|\bar{\zeta}_{p}\right|^{2 k}<C\left(1+\mathbb{E}\left|\zeta_{0}\right|^{2 k}\right) .
\end{gathered}
$$

(ii) There exists a positive constant $C$ such that, for any initial condition $y$,

$$
\mathbb{E}\left|\zeta_{h}-\bar{\zeta}_{1}\right|^{2} \leq C h^{3}
$$

and, for any $p=1, \ldots, M=\frac{T}{h}$,

$$
\mathbb{E}\left|\zeta_{p h}-\bar{\zeta}_{p}\right|^{2} \leq C h^{2}
$$

Remark 2.4. When $\sigma_{0}$ is not a constant function, for the Euler scheme, one generically has

$$
\mathbb{E}\left|\zeta_{p h}-\hat{\zeta}_{p}\right|^{2} \leq C h
$$

When $b_{0}$ and $\sigma_{0}$ are constant functions, there is no approximation error.

We now state some consequences of the hypotheses (H4) or (H5).

Remark 2.5. Under (H3), (H4), one has

$$
\begin{aligned}
\int_{0}^{+\infty} & u_{0}(x) d x+\int_{-\infty}^{0}\left(1-u_{0}(x)\right) d x \\
= & -\int_{0}^{+\infty} x d u_{0}(x)+\int_{-\infty}^{0} x d u_{0}(x) \\
& =\int_{\mathbf{R}}|x| d\left(1-u_{0}\right)(x)<+\infty
\end{aligned}
$$

The hypothesis (H5) instead of (H4) implies

$$
\int_{0}^{+\infty} u_{0}(x) d x+\int_{-\infty}^{0}\left(i-u_{0}(x)\right) d x \leq \sup _{i=1, \ldots, N}\left|\omega_{0}^{i}\right| \sum_{i=1}^{N}\left|x_{0}^{i}\right| .
$$

Lemma 2.6. Assume that $u_{0}$ satisfies hypotheses (H3), (H4); then there exists $C>0$ such that

$$
\int_{0}^{+\infty} u(t, x) d x+\int_{-\infty}^{0}(1-u(t, x)) d x \leq C
$$

for any $t \in[0, T]$. 
If $u_{0}$ satisfies $(\mathrm{H} 5)$, then

$$
\int_{0}^{+\infty} u(t, x) d x+\int_{-\infty}^{0}(1-u(t, x)) d x \leq C\left(1+\sup _{i=1, \ldots, N}\left|\omega_{0}^{i}\right| \sum_{i=1}^{N}\left|x_{0}^{i}\right|\right) .
$$

The proof is in the Supplement.

The next lemma gives a control of the error due to the permutation of the expectation and a nonlinear function, and simply follows from a Taylor expansion of $g(x)-g(\mathbb{E} X)$.

Lemma 2.7. Let $g$ be a function of class $C^{2}$ with bounded second derivative; then for any square integrable random variable $X$ we have

$$
|\mathbb{E} g(X)-g(\mathbb{E} X)| \leq C \mathbb{E}(X-\mathbb{E} X)^{2} .
$$

Finally, the next equality will be useful in several subsequent computations: for any $y, z \in \mathbb{R}$, there holds

$$
\int_{\mathbb{R}}|H(y-x)-H(z-x)| d x=|y-z| .
$$

\section{REPRESENTATION OF THE SOLUTION OF (1.1) AND CONSTRUCTION OF THE ALGORITHM}

3.1. A probabilistic representation of the solution. We introduce a probability space $(\Omega, \mathscr{F}, \mathbb{P})$ equipped with a Brownian motion $(B(t))$; for $0 \leq s<t \leq T$, $\mathscr{F}_{s}{ }^{t}$ will denote the least complete $\sigma$-field for which all the $B_{v}-B_{u} \quad(s \leq u<$ $v \leq t$ ) are measurable.

Theorem 3.1. Under $(\mathrm{H} 1)-(\mathrm{H} 3)$, if $u_{0}$ is of class $C_{b}^{\infty}(\mathbb{R})$, we have the following representation:

$$
u(t, x)=\mathbb{E}\left[H\left(X_{t}-x\right) \exp \left(\int_{0}^{t} f^{\prime} \circ u\left(s, X_{s}\right) d s\right)\right]
$$

where $\left(X_{t}\right)$ is the solution to

$$
d X_{t}=\sigma\left(X_{t}\right) d B_{t}-\left\{b\left(X_{t}\right)-\sigma\left(X_{t}\right) \sigma^{\prime}\left(X_{t}\right)\right\} d t .
$$

Here, the law of $X_{0}$ has a density equal to $-u_{0}^{\prime}$, and $\left(B_{t}\right)$ is a standard Brownian motion.

Proof. The function $v(t, x):=\frac{\partial u}{\partial x}(t, x)$ satisfies the following equation:

$$
\left\{\begin{aligned}
\frac{\partial v}{\partial t}(t, x)= & \frac{1}{2} \sigma^{2}(x) \frac{\partial^{2} v}{\partial x^{2}}(t, x)+\left(b(x)+\sigma(x) \sigma^{\prime}(x)\right) \frac{\partial v}{\partial x}(t, x) \\
& +\left(b^{\prime}(x)+f^{\prime} \circ u(t, x)\right) v(t, x), \\
v(0, x)= & u_{0}^{\prime}(x) .
\end{aligned}\right.
$$

By applying the Feynman-Kac formula, we obtain

$$
v(t, x)=\mathbb{E}\left[u_{0}^{\prime}\left(Y_{t}(x)\right) \exp \left\{\int_{0}^{t}\left[b^{\prime}\left(Y_{s}(x)\right)+f^{\prime} \circ u\left(t-s, Y_{s}(x)\right)\right] d s\right\}\right]
$$

where $\left(Y_{t}\right)$ is the solution to

$$
d Y_{t}=\left(b\left(Y_{t}\right)+\sigma\left(Y_{t}\right) \sigma^{\prime}\left(Y_{t}\right)\right) d t+\sigma\left(Y_{t}\right) d B_{t}
$$


Consequently, recalling that $1-u_{0}$ is increasing and that $u(t, x) \rightarrow 1$ as $x \rightarrow-\infty$ (see Lemma 2.6), we have

$$
u(t, x)=-\mathbb{E} \int_{x}^{+\infty} u_{0}^{\prime}\left(Y_{t}(y)\right) \exp \left\{\int_{0}^{t}\left[b^{\prime}\left(Y_{s}(y)\right)+f^{\prime} \circ u\left(t-s, Y_{s}(y)\right)\right] d s\right\} d y .
$$

As we will see below, the particles algorithm is based on approximating the measure $u_{0}^{\prime}(y) d y$ by a measure of type $\sum_{i=1}^{N} \omega_{0}^{i} \delta_{x_{0}^{i}}$; that suggests one performs the change of variable $z=\xi_{0, t}(y)$, where $\xi_{0, t}(\cdot)$ is the flow associated with the stochastic differential equation (3.4). Hence, we set $y=\xi_{0, t}^{-1}(z)$.

Using results of the second chapter of Kunita [8], we have, for $\theta<t$,

$$
\xi_{\theta, t}^{-1}(z)=z-\int_{\theta}^{t} \sigma\left(\xi_{s, t}^{-1}(z)\right) \hat{d} B_{s}-\int_{\theta}^{t} b\left(\xi_{s, t}^{-1}(z)\right) d s,
$$

where $\hat{d} B_{\theta}$ denotes the "backward" stochastic integral. ${ }^{3}$

One infers that

$$
\frac{\partial}{\partial z} \xi_{0, t}^{-1}(z)=1-\int_{0}^{t} \sigma^{\prime}\left(\xi_{\theta, t}^{-1}(z)\right) \frac{\partial}{\partial z} \xi_{\theta, t}^{-1}(z) \hat{d} B_{\theta}-\int_{0}^{t} b^{\prime}\left(\xi_{\theta, t}^{-1}(z)\right) \frac{\partial}{\partial z} \xi_{\theta, t}^{-1}(z) d \theta,
$$

from which

$$
\frac{\partial}{\partial z} \xi_{0, t}^{-1}(z)=\exp \left(\int_{0}^{t}\left\{-b^{\prime}\left(\xi_{\theta, t}^{-1}(z)\right)-\frac{1}{2} \sigma^{\prime 2}\left(\xi_{\theta, t}^{-1}(z)\right)\right\} d \theta-\int_{0}^{t} \sigma^{\prime}\left(\xi_{\theta, t}^{-1}(z)\right) \hat{d} B_{\theta}\right) .
$$

Hence, taking into account (2.5), we have

$$
\begin{aligned}
& u(t, x)=-\mathbb{E} {\left[\int_{\xi_{0, t}(x)}^{+\infty} u_{0}^{\prime}(z)\right.} \\
& \cdot \exp \left\{\left.\int_{0}^{t}\left(b^{\prime}\left(\xi_{0, s}(\alpha)\right)+f^{\prime} \circ u\left(t-s, \xi_{0, s}(\alpha)\right)\right) d s\right|_{\alpha=\xi_{0, t}^{-1}(z)}\right\} \\
& \cdot \exp \left\{\int_{0}^{t}\left[-b^{\prime}\left(\xi_{s, t}^{-1}(z)\right)-\frac{1}{2} \sigma^{\prime 2}\left(\xi_{s, t}^{-1}(z)\right)\right] d s\right. \\
&\left.\left.-\int_{0}^{t} \sigma^{\prime}\left(\xi_{s, t}^{-1}(z)\right) \hat{d} B_{s}\right\} d z\right] .
\end{aligned}
$$

One now uses Lemma 6.2 of Chapter II of Kunita [8]: for any continuous function $g(s, x)$ we have

$$
\left.\int_{0}^{t} g\left(s, \xi_{0, s}(\alpha)\right)\right|_{\alpha=\xi_{0, t}^{-1}(z)} d s=\int_{0}^{t} g\left(s, \xi_{s, t}^{-1}(z)\right) d s .
$$

Thus,

$$
\begin{aligned}
& u(t, x)=-\mathbb{E}\left[\int_{-\infty}^{+\infty} H\left(-\xi_{0, t}(x)+z\right)\right. \\
&\left.\cdot \exp \left\{\int_{0}^{t} f^{\prime} \circ u\left(t-s, \xi_{s, t}^{-1}(z)\right) d s\right\} M_{0}^{t}(z) u_{0}^{\prime}(z) d z\right],
\end{aligned}
$$

\footnotetext{
${ }^{3}$ For a definition, cf. Kunita [8, end of Chapter I].
} 
where $\left(M_{\theta}^{t}(z)\right)_{\theta \leq t}$ is the exponential (backward) $\left(\mathscr{F}_{\theta}^{t}\right)_{\theta \leq t}$-martingale defined by

$$
M_{\theta}^{t}(z)=\exp \left\{-\frac{1}{2} \int_{\theta}^{t} \sigma^{\prime 2}\left(\xi_{s, t}^{-1}(z)\right) d s-\int_{\theta}^{t} \sigma^{\prime}\left(\xi_{s, t}^{-1}(z)\right) \hat{d} B_{s}\right\} .
$$

The application $x \rightarrow \xi_{0, t}(x)$ is a.s. increasing (its derivative is an exponential); thus $H\left(-\xi_{0, t}(x)+z\right)=H\left(\xi_{0, t}^{-1}(z)-x\right)$.

Hence,

$$
u(t, x)=\mathbb{E}\left[\int_{-\infty}^{+\infty} H\left(\xi_{0, t}^{-1}(z)-x\right) \exp \left\{\int_{0}^{t} f^{\prime} \circ u\left(s, \xi_{t-s, t}^{-1}(z)\right) d s\right\} M_{0}^{t}(z) d z\right] .
$$

We observe that the law of the process $\left(\xi_{t-\theta, t}^{-1}\right)_{0 \leq \theta \leq t}$, on $\left(\Omega, \mathscr{F}, \mathbb{P}, \mathscr{F}_{0}^{t}\right)$, is identical to the law of the process $\left(X_{\theta}\right)_{0 \leq \theta \leq t}$, solution to

$$
d X_{\theta}=\sigma\left(X_{\theta}\right) d B_{\theta}-b\left(X_{\theta}\right) d \theta .
$$

Hence, $\mathbb{E}_{0}$ denoting the expectation under the law $\mathbb{P}_{0}$ for which the initial law of the process $\left(X_{\theta}\right)$ has a density equal to $-u_{0}^{\prime}(z)$, and $\left(M_{t}\right)$ denoting the exponential martingale defined by

$$
M_{t}=\exp \left\{-\frac{1}{2} \int_{0}^{t} \sigma^{\prime 2}\left(X_{s}\right) d s+\int_{0}^{t} \sigma^{\prime}\left(X_{s}\right) d B_{s}\right\}
$$

we have

$$
u(t, x)=\mathbb{E}_{0}\left[H\left(X_{t}-x\right) \exp \left\{\int_{0}^{t} f^{\prime} \circ u\left(t-s, X_{s}\right) d s\right\} M_{t}\right] .
$$

On $\left(\Omega, \mathscr{F}, \mathbb{P}_{0}, \mathscr{F}_{0}{ }^{T}\right)$, one performs the Girsanov transformation defined by

$$
\widetilde{\mathbb{P}}(A):=\mathbb{E}_{0}\left[1_{A} M_{T}\right], \quad A \in \mathscr{F}_{0}{ }^{T} ;
$$

then, for $t \leq T$,

$$
u(t, x)=\widetilde{\mathbb{E}}\left[H\left(X_{t}-x\right) \exp \left\{\int_{0}^{t} f^{\prime} \circ u\left(s, X_{s}\right) d s\right\}\right] .
$$

Under $\widetilde{\mathbb{P}}$, the process $\left(X_{t}\right)$ solves

$$
d X_{t}=\sigma\left(X_{t}\right) d \widetilde{B}_{t}-\left\{b\left(X_{t}\right)-\sigma\left(X_{t}\right) \sigma^{\prime}\left(X_{t}\right)\right\} d t .
$$

Here, $\left(\widetilde{B}_{\theta}\right)$ defined by

$$
\widetilde{B}_{\theta}=B_{\theta}-\int_{0}^{\theta} \sigma^{\prime}\left(X_{s}\right) d s
$$

is a Brownian motion under $\widetilde{\mathbb{P}}$. Obviously, the above representation of $u$ is identical to (3.1).

One can deduce a result of the same type as the preceding one for a piecewise constant initial data $u_{0}$ :

Proposition 3.2. If $u_{0}$ is of the form $\sum_{i=1}^{N} \omega_{0}^{i} H\left(x_{0}^{i}-x\right)$, then we have

$$
u(t, x)=\sum_{i=1}^{N} \omega_{0}^{i} \mathbb{E}\left[H\left(X_{t}\left(x_{0}^{i}\right)-x\right) \exp \left\{\int_{0}^{t} f^{\prime} \circ u\left(s, X_{s}\left(x_{0}^{i}\right)\right) d s\right\}\right] .
$$

The proof (based upon an approximation argument) is in the Supplement. 
3.2. Principle of the algorithm. Let $T>0$ be fixed, and $h$ a time discretization step of type $\frac{T}{M}$, for some integer $M$. We want to approximate $u(T, x)$. Approximating $-u_{0}^{\prime}(z) d z$ by $\sum_{i=1}^{N} \omega_{0}^{i} \delta_{x_{0}^{i}}$, one gets the following approximation formula:

$$
u(T, x) \simeq \sum_{i=1}^{N} \omega_{0}^{i} \mathbb{E}\left[H\left(X_{T}\left(x_{0}^{i}\right)-x\right) \exp \left\{\int_{0}^{T} f^{\prime} \circ u\left(s, X_{s}\left(x_{0}^{i}\right)\right) d s\right\}\right] .
$$

Now, on $(\Omega, \mathscr{F}, \mathbb{P})$, we are given $N$ independent Brownian motions $\left\{\left(B_{\theta}^{i}\right)\right.$, $i=1, \ldots, N\}$ with respect to the filtration $\left(\mathscr{F}_{0}^{\theta}\right)_{\theta>0}$, simply denoted by $\left(\mathscr{G}_{\theta}\right)$ in the sequel.

Let $\left(X_{\theta}^{i}\right)$ be the (independent) solutions to the following SDEs (in forward time):

$$
\left\{\begin{array}{l}
d X_{\theta}^{i}=\sigma\left(X_{\theta}^{i}\right) d B_{\theta}^{i}-\left\{b\left(X_{\theta}^{i}\right)-\sigma\left(X_{\theta}^{i}\right) \sigma^{\prime}\left(X_{\theta}^{i}\right)\right\} d \theta, \\
X_{0}^{i}=x_{0}^{i} .
\end{array}\right.
$$

The particle algorithm replaces the expectation by a point estimation:

$$
u(T, x) \simeq \sum_{i=1}^{N} \omega_{0}^{i} H\left(X_{T}^{i}-x\right) \exp \left\{\int_{0}^{T} f^{\prime} \circ u\left(s, X_{s}^{i}\right) d s\right\} .
$$

Then we approximate

$$
\exp \left\{\int_{0}^{T} f^{\prime} \circ u\left(s, X_{s}^{i}\right) d s\right\} \quad \text { by } \exp \left\{h \sum_{0}^{M-1} f^{\prime} \circ u\left(p h, X_{p h}^{i}\right)\right\},
$$

and if we define by induction

$$
\rho_{0}^{i}=\omega_{0}^{i}, \quad \rho_{(k+1) h}^{i}=\rho_{k h}^{i} \exp \left\{h f^{\prime} \circ u\left(k h, X_{k h}^{i}\right)\right\},
$$

we get, for any $p=0,1, \ldots, M=T / h$,

$$
u(p h, x) \simeq \sum_{i=1}^{N} \rho_{p h}^{i} H\left(X_{p h}^{i}-x\right) .
$$

In fact, the $\left(X_{p h}^{i}\right)$ 's will be, in turn, approximated by the Milshtein scheme (2.7) applied to (3.2):

$$
\begin{aligned}
\bar{X}_{p+1}^{i}= & \bar{X}_{p}^{i}-\left(b\left(\bar{X}_{p}^{i}\right)-\sigma\left(\bar{X}_{p}^{i}\right) \sigma^{\prime}\left(\bar{X}_{p}^{i}\right)\right) h+\sigma\left(\bar{X}_{p}^{i}\right)\left(B_{(p+1) h}^{i}-B_{p h}^{i}\right) \\
& +\frac{1}{2} \sigma\left(\bar{X}_{p}^{i}\right) \sigma^{\prime}\left(\bar{X}_{p}^{i}\right)\left(\left(B_{(p+1) h}^{i}-B_{p h}^{i}\right)^{2}-h\right) .
\end{aligned}
$$

Thus, if we define

$$
\bar{\rho}_{0}^{i}=\omega_{0}^{i}, \quad \bar{\rho}_{(k+1) h}^{i}=\bar{\rho}_{k h}^{i} \exp \left\{h f^{\prime} \circ u\left(k h, \bar{X}_{k}^{i}\right)\right\},
$$

we have

$$
u(p h, x) \simeq \sum_{i=1}^{N} \bar{\rho}_{p h}^{i} H\left(\bar{X}_{p}^{i}-x\right) .
$$

Actually, one considers the weights in a slightly different way in order that the sum of the weights is equal to 1 (this fact will be used in the sequel):

$$
\begin{aligned}
\bar{\rho}_{k h}^{i} \exp \left\{h f^{\prime} \circ u\left(k h, \bar{X}_{k}^{i}\right\}\right. & \simeq \bar{\rho}_{k h}^{i}+h f^{\prime} \circ u\left(k h, \bar{X}_{k}^{i}\right) \bar{\rho}_{k h}^{i} \\
& \simeq \bar{\rho}_{k h}^{i}+h\left(f \circ u\left(k h, \bar{X}_{k}^{i}\right)-f \circ u\left(k h, \bar{X}_{k}^{\pi_{k}(i)}\right)\right),
\end{aligned}
$$


where $\pi_{k}(i)$ denotes the label number of the particle just to the right of the particle of label $i$ at the time $k h$ (if the considered particle is at the rightmost position at time $k h$, we just have $\bar{\rho}_{k h}^{i} \exp \left\{h f^{\prime} \circ u\left(k h, \bar{X}_{k}^{i}\right\} \simeq \bar{\rho}_{k h}^{i}+h f \circ u\left(k h, \bar{X}_{k}^{i}\right)\right)$. That transformation of the weights corresponds to the step " $R_{k \Delta t}$ " of the splitting method of Puckett (cf. [12]).

3.3. The algorithm. Finally, the algorithm will be the following: we define the initial weights and the initial approximation by

$$
\omega_{0}^{i}=\frac{1}{N} \quad \text { for } i=1, \ldots, N ; \quad \bar{u}_{0}(x)=\sum_{i=1}^{N} \omega_{0}^{i} H\left(x_{0}^{i}-x\right),
$$

where

$$
\forall i<N: \quad x_{0}^{i}=u_{0}^{-1}\left(1-\frac{i}{N}\right), \quad x_{0}^{N}=u_{0}^{-1}\left(\frac{1}{2 N}\right) .
$$

Evidently, $\bar{u}_{0}(\cdot)$ is a piecewise constant approximation to the initial datum $u_{0}(\cdot)$.

Recall that we define the approximating process by (3.6).

We now define, in a recursive way (and using the same convention for the particle at the rightmost position as previously):

$$
\omega_{p}^{i}=\omega_{p-1}^{i}\left(1+h \frac{f \circ \bar{u}_{p-1}\left(\bar{X}_{p-1}^{i}\right)-f \circ \bar{u}_{p-1}\left(\bar{X}_{p-1}^{\pi_{p-1}(i)}\right)}{\omega_{p-1}^{i}}\right)
$$

and

$$
\bar{u}_{p}(x)=\sum_{i=1}^{N} \omega_{p}^{i} H\left(\bar{X}_{p}^{i}-x\right)
$$

for $p=1,2, \ldots, M=T / h$.

Remark 3.3. All the weights, for some constant $C$ uniform in $h, N, i$, and $k$, are bounded by

$$
0 \leq \omega_{k}^{i} \leq \frac{C}{N}
$$

and, for any $k=1, \ldots, M=T / h$, the weights $\omega_{k}^{i}(i=1, \ldots, N)$ are $\mathscr{G}_{(k-1) h^{-}}$ measurable (this will play an important role in the sequel). Moreover, it is easy to check from the definition (3.8) that, for any $p=1, \ldots, M=T / h$,

$$
\sum_{i=1}^{N} \omega_{p}^{i}=1
$$

By using the fact that $f^{\prime}$ and $f^{\prime \prime}$ are bounded, and that the $\omega_{p h}$ 's are bounded by $C / N$, we have

$$
\omega_{p}^{i}=\omega_{p-1}^{i}\left(1+h f^{\prime} \circ \bar{u}_{p-1}\left(\bar{X}_{p-1}^{i}\right)\right)+\mathscr{O}(h) \mathscr{O}\left(\frac{1}{N^{2}}\right) .
$$

Proposition 3.4. Under the hypotheses $(\mathrm{H} 1)-(\mathrm{H} 4)$, we have

$$
\frac{1}{N} \sum_{i=1}^{N}\left|x_{0}^{i}\right|^{2} \leq C \text {. }
$$


In addition,

$$
\frac{1}{N} \sum_{i=1}^{N} \mathbb{E}\left|\bar{X}_{p}^{i}\right|^{2} \leq C .
$$

The proof (which uses (2.8)) is in the Supplement.

\section{THE MAIN RESUlT}

The main result of the paper is the following theorem.

Theorem 4.1. (i) Under the hypotheses $(\mathrm{H} 1)-(\mathrm{H} 4)$, there exist strictly positive constants $C$ and $h_{0}<1$ such that, for any $h<h_{0}$ and any $N \geq 1$,

$$
\|u(T, \cdot)-\bar{u}(T, \cdot)\|_{L^{2}(\mathbf{R} \times \Omega)} \leq C\left(\frac{1}{\sqrt{N}}+\sqrt{h}\right) .
$$

(ii) When the functions $b$ and $\sigma$ are constant, then the rate of convergence is given by

$$
\|u(T, \cdot)-\bar{u}(T, \cdot)\|_{L^{1}(\mathbf{R} \times \Omega)} \leq C\left(\frac{1}{\sqrt{N}}+h\right) .
$$

The same estimates hold for the standard deviation of $\|u(T, \cdot)-\bar{u}(T, \cdot)\|_{L^{1}(\mathbf{R})}$.

When $f \equiv 0$, the estimate (i) can be improved. Indeed, if $\mu_{0}$ denotes the probability measure whose $1-u_{0}$ is the cumulative function, and $\left(X_{t}\right)$ is defined by

$$
d X_{t}=\sigma\left(X_{t}\right) d B_{t}-\left\{b\left(X_{t}\right)-\sigma\left(X_{t}\right) \sigma^{\prime}\left(X_{t}\right)\right\} d t,
$$

then, from (3.1), $u(t, x)=\mathbb{E}_{\mu_{0}} H\left(X_{t}-x\right)$ and to the error

$$
\|u(T, \cdot)-\bar{u}(T, \cdot)\|_{L^{1}(\mathbf{R} \times \Omega)}
$$

contribute a statistical error

$$
\left\|u(T, \cdot)-\frac{1}{N} H\left(X_{T}^{i}-\cdot\right)\right\|_{L^{1}(\mathbb{R} \times \Omega)},
$$

which is of order $1 / \sqrt{N}$, and an approximation error

$$
\left\|\frac{1}{N} H\left(X_{T}^{i}-\cdot\right)-\frac{1}{N} H\left(\bar{X}_{T}^{i}-\cdot\right)\right\|_{L^{1}(\mathbf{R} \times \Omega)},
$$

which generically is of order $h$ when the Milshtein scheme is used. The nonlinearity of the PDE induced by $f$ changes the order of convergence, at least in our proofs. Our numerical experiments have not permitted us to check whether $\sqrt{h}$ is the best estimate: typically, the algorithm was extremely sensitive to $h$; when $h$ was small, it was difficult to isolate the error due to the discretization from the statistical error (we could not choose $N$ so large as it would have been necessary), and for different, but not small $h$, some numerical instabilities produced statistical and discretization errors of comparable magnitude. In any case, the important point seems to us that the behavior of the error can be described without assuming a relation between $h$ and $N$.

The gain in accuracy, when $b$ and $\sigma$ are constant, is not mysterious: to give a feeling of what happens, suppose $b \equiv 0$ and $\sigma \equiv 1$; in that case, the particles are Brownian, and the law of the $X_{(p+1) h}^{i}-X_{p h}^{i}$ 's can be simulated exactly (one 
just has to simulate independent Gaussian variables), whereas, when $\sigma$ is not constant, one has to approximate the processes $\left(X_{t}^{i}\right)$; the passage from $h$ to $\sqrt{h}$ is due to this approximation (see Proposition 2.3).

We also remark that, when the coefficients are not constant, we obtained the above estimates after having used the Milshtein scheme, not the Euler scheme (compare Remark 2.11 and inequality (2.10)). Finally, we stress that the Euler and Milshtein schemes are the only schemes reasonable from the point of view of numerical efficiency (see Talay [16]).

The next three sections are devoted to the proof of part (i) of this theorem. In $\S 8$, we will explain what must be changed in the proof in order to obtain the better estimate in part (ii). Similar computations permit us to obtain the estimates for the standard deviation.

\section{THE WEIGHTS ARE NOT FAR FROM BEING INDEPENDENT}

The $\omega_{p}^{i}$ 's are not independent, but we can choose other weights that are independent and approximate the $\omega_{p}^{i}$ 's in order to get, in the sequel, useful estimates.

We define $\rho_{p}^{i}$ by

$$
\rho_{0}^{i}=\omega_{0}^{i}, \quad \rho_{p}^{i}=\rho_{p-1}^{i}\left(1+h f^{\prime} \circ u\left((p-1) h, X_{(p-1) h}^{i}\right)\right) .
$$

The $\rho_{p}^{i}$ 's $(i=1, \ldots, N)$ are independent, and it is easy to show there exists a $C>0$ such that $\left|\rho_{p}^{i}\right| \leq \frac{C}{N}$.

Set $\alpha_{p}^{i}:=\mathbb{E}\left|\omega_{p}^{i}-\rho_{p}^{i}\right|^{2}$, and $\alpha_{p}:=\sup _{i} \alpha_{p}^{i}$.

The objective of this section is to prove (cf. Proposition 5.8)

$$
\forall h, \quad \forall p=1, \ldots, M=\frac{T}{h}: \quad \alpha_{p} \leq \frac{C h}{N^{2}}+\frac{C}{N^{3}} .
$$

Remark 5.1. We observe

$$
\begin{aligned}
\omega_{p+1}^{i}-\rho_{p+1}^{i}= & \omega_{p}^{i}-\rho_{p}^{i}+h \omega_{p}^{i}\left\{f^{\prime} \circ \bar{u}_{p}\left(\bar{X}_{p}^{i}\right)-f^{\prime} \circ u\left(p h, \bar{X}_{p}^{i}\right)\right\} \\
& +h\left(\omega_{p}^{i}-\rho_{p}^{i}\right) f^{\prime} \circ u\left(p h, \bar{X}_{p}^{i}\right) \\
& +h \rho_{p}^{i}\left\{f^{\prime} \circ u\left(p h, \bar{X}_{p}^{i}\right)-f^{\prime} \circ u\left(p h, X_{p h}^{i}\right)\right\}+\mathscr{O}\left(\frac{h}{N^{2}}\right) .
\end{aligned}
$$

As $f^{\prime} \circ u$ is Lipschitz, and as $\bar{X}$ is defined by the Milshtein scheme (cf. Proposition 2.3), we get

$$
\alpha_{p+1}^{i} \leq \alpha_{p}^{i}+\frac{C h}{N} \sqrt{\alpha_{p}^{j}} \sqrt{\mathbb{E}\left|\bar{u}_{p}\left(\bar{X}_{p}^{i}\right)-u\left(p h, \bar{X}_{p}^{i}\right)\right|^{2}}+C h \alpha_{p}^{i}+C \frac{h^{2}}{N^{2}}+C \frac{h}{N^{3}} .
$$

We now need to get a precise estimate of $\sqrt{\mathbb{E}\left|\bar{u}_{p}\left(\bar{X}_{p}^{i}\right)-u\left(p h, \bar{X}_{p}^{i}\right)\right|^{2}}$. Having defined

$$
u_{p}^{*}(x)=\sum_{j=1}^{N} \rho_{p}^{j} H\left(X_{p h}^{j}-x\right),
$$

we have

$$
\begin{aligned}
& \sqrt{\mathbb{E}\left|\bar{u}_{p}\left(\bar{X}_{p}^{i}\right)-u\left(p h, \bar{X}_{p}^{i}\right)\right|^{2}} \\
& \quad \leq \sqrt{\mathbb{E}\left|\bar{u}_{p}\left(\bar{X}_{p}^{i}\right)-u_{p}^{*}\left(\bar{X}_{p}^{i}\right)\right|^{2}}+\sqrt{\mathbb{E}\left|u_{p}^{*}\left(\bar{X}_{p}^{i}\right)-u\left(p h, \bar{X}_{p}^{i}\right)\right|^{2}}
\end{aligned}
$$


An upper bound for the first term of the right-hand side will be given in $\S 5.1$ below, an upper bound for the second term in $\S 5.2$, and finally we will come back to the inequality $(5.2)$ in $\S 5.3$.

5.1. An upper bound for $\mathbb{E}\left|\bar{u}_{p}\left(\bar{X}_{p}^{i}\right)-u_{p}^{*}\left(\bar{X}_{p}^{i}\right)\right|^{2}$.

Propositoin 5.2. There holds

$$
\mathbb{E}\left|\bar{u}_{p}\left(\bar{X}_{p}^{i}\right)-u_{p}^{*}\left(\bar{X}_{p}^{i}\right)\right|^{2} \leq 2 N^{2} \alpha_{p}+\frac{C}{N}+C h .
$$

Proof. We have

$$
\begin{aligned}
& \mathbb{E}\left|\bar{u}_{p}\left(\bar{X}_{p}^{i}\right)-u_{p}^{*}\left(\bar{X}_{p}^{i}\right)\right|^{2} \\
& \leq 2 \mathbb{E}\left[\sum_{j=1}^{N}\left|\omega_{p}^{j}-\rho_{p}^{j}\right| H\left(\bar{X}_{p}^{j}-\bar{X}_{p}^{i}\right)\right]^{2} \\
& +2 \mathbb{E}\left[\sum_{j=1}^{N} \rho_{p}^{j}\left|H\left(\bar{X}_{p}^{j}-\bar{X}_{p}^{i}\right)-H\left(X_{p h}^{j}-\bar{X}_{p}^{i}\right)\right|\right]^{2} \\
& \leq 2 \sum_{j, k=1}^{N} \sqrt{\alpha_{p}^{j} \alpha_{p}^{k}}+\frac{C}{N^{2}} \sum_{j=1}^{N} \mathbb{E}\left|H\left(\bar{X}_{p}^{j}-\bar{X}_{p}^{i}\right)-H\left(X_{p h}^{j}-\bar{X}_{p}^{i}\right)\right|^{2} \\
& +\frac{C}{N_{2}} \sum_{\substack{j, k=1 \\
j \neq k \\
j \neq i, k \neq i}}^{N} \mathbb{E}\left|H\left(\bar{X}_{p}^{j}-\bar{X}_{p}^{i}\right)-H\left(X_{p h}^{j}-\bar{X}_{p}^{i}\right)\right| \\
& \cdot\left|H\left(\bar{X}_{p}^{k}-\bar{X}_{p}^{i}\right)-H\left(X_{p h}^{k}-\bar{X}_{p}^{i}\right)\right|+\frac{C}{N} \\
& \leq 2 \sum_{j, k=1}^{N} \sqrt{\alpha_{p}^{j} \alpha_{p}^{k}}+\frac{C}{N} \\
& +\frac{C}{N^{2}} \sum_{\substack{j, k=1 \\
j \neq k \\
j \neq i, k \neq i}}^{N} \mathbb{E}\left|H\left(\bar{X}_{p}^{j}-\bar{X}_{p}^{i}\right)-H\left(X_{p h}^{j}-\bar{X}_{p}^{i}\right)\right| \\
& \cdot\left|H\left(\bar{X}_{p}^{k}-\bar{X}_{p}^{i}\right)-H\left(X_{p h}^{k}-\bar{X}_{p}^{i}\right)\right| .
\end{aligned}
$$

Therefore, to get the conclusion it remains to prove

Lemma 5.3. For $i \neq j \neq k$ one has

$$
\mathbb{E}\left|H\left(\bar{X}_{p}^{j}-\bar{X}_{p}^{i}\right)-H\left(X_{p h}^{j}-\bar{X}_{p}^{i}\right)\right| \cdot\left|H\left(\bar{X}_{p}^{k}-\bar{X}_{p}^{i}\right)-H\left(X_{p h}^{k}-\bar{X}_{p}^{i}\right)\right| \leq C h .
$$

The proof is in the Supplement.

5.2. An upper bound for $\mathbb{E}\left|u_{p}^{*}\left(\bar{X}_{p}^{i}\right)-u\left(p h, \bar{X}_{p}^{i}\right)\right|^{2}$. For brevity, we will denote

$$
U_{p}^{i}:=\mathbb{E}\left|u_{p}^{*}\left(\bar{X}_{p}^{i}\right)-u\left(p h, \bar{X}_{p}^{i}\right)\right|^{2} .
$$

The objective of this subsection is to prove (see (10.4) in the Supplement) 
Proposition 5.4. There holds

$$
U_{p}^{i} \leq \frac{C}{N}+C h^{2}
$$

Proof. Let $u^{N}(t, x):=\sum_{j=1}^{N} \omega_{0}^{j} \mathbb{E}\left[H\left(X_{t}^{j}-x\right) \exp \left(\int_{0}^{t} f^{\prime} \circ u\left(s, X_{s}^{j}\right) d s\right)\right]$. Then

$$
\begin{aligned}
& U_{p}^{i} \leq C \mathbb{E}\left|u\left(p h, \bar{X}_{p}^{i}\right)-u^{N}\left(p h, \bar{X}_{p}^{i}\right)\right|^{2} \\
&+ C \mathbb{E}\left\{\sum_{j=1}^{N} H\left(X_{p h}^{j}-\bar{X}_{p}^{i}\right)\left[\omega_{0}^{j} e^{\int_{0}^{p h} f^{\prime} \circ u\left(s, X_{s}^{j}\right) d s}-\rho_{p}^{j}\right]\right\}^{2} \\
&+\left\{\mathbb { E } \left\{\sum _ { j = 1 } ^ { N } \omega _ { 0 } ^ { j } \left[\mathbb{E} H\left(X_{p h}^{j}-\bar{X}_{p}^{i}\right) e^{\int_{0}^{p h} f^{\prime} \circ u\left(s, X_{s}^{j}\right) d s}\right.\right.\right. \\
&\left.\left.-H\left(X_{p h}^{j}-\bar{X}_{p}^{i}\right) e^{\int_{0}^{p h} f^{\prime} \circ u\left(s, X_{s}^{j}\right) d s}\right]\right\}^{2} .
\end{aligned}
$$

Each of the three following lemmas will deal with a term of the right-hand side of the preceding inequality. The proofs are in the Supplement.

Lemma 5.5. There exists a $C>0$ such that for any $t \in[0, T]$ we have

$$
\left\|u(t, \cdot)-u^{N}(t, \cdot)\right\|_{L^{\infty}(\mathbf{R})} \leq \frac{C}{N} .
$$

Lemma 5.6. There holds

$$
T_{p}^{N}:=\mathbb{E}\left|\sum_{j=1}^{N} H\left(X_{p h}^{j}-\bar{X}_{p}^{i}\right)\left\{\omega_{0}^{j} \exp \int_{0}^{p h} f^{\prime} \circ u\left(s, X_{s}^{j}\right) d s-\rho_{p}^{j}\right\}\right|^{2} \leq C h^{2} .
$$

Lemma 5.7. There holds

$$
S_{p}^{N}:=\mathbb{E}\left|u^{N}\left(p h, \bar{X}_{p}^{i}\right)-\sum_{j=1}^{N} \omega_{0}^{j} H\left(X_{p h}^{j}-\bar{X}_{p}^{i}\right) \exp \int_{0}^{p h} f^{\prime} \circ u\left(s, X_{s}^{j}\right) d s\right|^{2} \leq \frac{C}{N} .
$$

5.3. An upper bound for $\alpha_{p}$. From the two previous subsections, we obtain, considering (5.2), (5.3), (5.4), and (5.5),

$$
\alpha_{p+1} \leq \alpha_{p}+\frac{C h}{N} \sqrt{\alpha_{p}}\left(N \sqrt{\alpha_{p}}+\frac{1}{\sqrt{N}}+\sqrt{h}\right)+C h \alpha_{p}+\frac{C h^{2}}{N^{2}}+\frac{C h}{N^{3}} .
$$

Proposition 5.8. We have, for all $p$,

$$
\alpha_{p} \leq \frac{C h}{N^{2}}+\frac{C}{N^{3}} .
$$

Proof. We have

$$
\alpha_{p+1} \leq(1+C h) \alpha_{p}+\frac{C h}{N} \sqrt{\alpha_{p}}\left(\frac{1}{\sqrt{N}}+\sqrt{h}\right)+\frac{C h^{2}}{N^{2}}+\frac{C h}{N^{3}} .
$$

We define by induction $\tau_{0}=\alpha_{0}$ and

$$
\tau_{p+1}=(1+C h) \tau_{p}+\frac{C h}{N} \sqrt{\tau_{p}}\left(\frac{1}{\sqrt{N}}+\sqrt{h}\right)+\frac{C h^{2}}{N^{2}}+\frac{C h}{N^{3}} .
$$


We observe that, for any $p$, there holds $\alpha_{p} \leq \tau_{p}$.

If, for any $p$, we have $\sqrt{\tau_{p}} \leq \frac{\sqrt{h}}{N}+\frac{1}{N \sqrt{N}}$, then we have obtained (5.6); otherwise, there exists a $j$ such that

$$
\left\{\begin{array}{l}
\sqrt{\tau_{j+1}}>\frac{\sqrt{h}}{N}+\frac{1}{N \sqrt{N}} \\
\sqrt{\tau_{j}} \leq \frac{\sqrt{h}}{N}+\frac{1}{N \sqrt{N}}
\end{array}\right.
$$

As $\left(\tau_{p}\right)$ is increasing, we would then have that, for any $p>j, \sqrt{\tau_{p}}>\frac{\sqrt{h}}{N}+$ $\frac{1}{N \sqrt{N}}$; then, for any $p>j$, we would also have

$$
\tau_{p+1} \leq(1+C h) \tau_{p}+C h \tau_{p}+\frac{C h^{2}}{N^{2}}+\frac{C h}{N^{3}},
$$

from which we deduce that

$$
\tau_{M} \leq(1+C h)^{M-j} \tau_{j}+\left(\frac{C h^{2}}{N^{2}}+\frac{C h}{N^{3}}\right) \frac{(1+C h)^{M-j}-1}{C h} \leq \frac{C h}{N^{2}}+\frac{C}{N^{3}} .
$$

Hence, (5.6) is true for any $p$.

\section{LOCAL EXPANSION OF THE SOLUTION $u(t, x)$}

For the sequel we need to compare the solution $u(t, x)$ to problem $(2.1)$ with the solution $v(t, x)$ to the problem

$$
\left\{\begin{array}{l}
\frac{\partial v}{\partial t}=L v \\
v(0, \cdot)=u_{0}(\cdot)
\end{array}\right.
$$

for small values of $t$. We can represent

$$
v(t, x)=\mathbb{E}\left(u_{0}\left(Z_{t}(x)\right)\right),
$$

where $\left(Z_{t}(x)\right)$ is the solution to the following equation:

$$
d Z_{t}=b\left(Z_{t}\right) d t+\sigma\left(Z_{t}\right) d B_{t}, \quad Z_{0}(x)=x .
$$

Let $P_{\theta}(x, d y)$ be the transition probability associated with $\left(Z_{t}\right)$.

Theorem 6.1. Assume the hypotheses (H1)-(H3); then for any $0<h<1$ and any $x \in \mathbb{R}$, we have

$$
u(h, x)=\mathbb{E} u_{0}\left(Z_{h}(x)\right)+h f\left(\mathbb{E} u_{0}\left(Z_{h}(x)\right)\right)+R_{h}(x)
$$

with the following estimate:

- if $u_{0}$ satisfies $(\mathrm{H} 4)$, then

$$
\left\|R_{h}(\cdot)\right\|_{L^{1}(\mathbb{R})} \leq C h^{2}
$$

- if $u_{0}$ belongs to a family of functions satisfying (H5) with weights bounded by $\frac{C}{N}$, the constant $C$ being uniform on the family, then

$$
\left\|R_{h}(\cdot)\right\|_{L^{1}(\mathbf{R})} \leq C h \sqrt{h}+C \frac{h^{2}}{N} \sum_{i=1}^{N}\left|x_{0}^{i}\right| .
$$

The proof is obtained by combining the propositions of this section and Remark 6.6: Proposition 6.3 expands $u(h, x)$, the others give estimates of the norm of the remaining terms in $L^{1}(\mathbb{R})$. 
Remark 6.2. The proof will make it clear that the constant in (6.4) can be made more explicit (see the footnotes in the sequel):

$$
\begin{aligned}
\left\|R_{h}(\cdot)\right\|_{L^{1}(\mathbf{R})} \leq & C h \sqrt{h}\left(\frac{1}{N}+\frac{C}{N^{2}} \sum_{i, j, i<j} \exp \left(-\frac{\left(x_{0}^{i}-x_{0}^{j}\right)^{2}}{8 \lambda h}\right)\right) \\
& +C \frac{h^{2}}{N} \sum_{i=1}^{N}\left|x_{0}^{i}\right| .
\end{aligned}
$$

This will be used to treat the special case of constant $b$ and $\sigma$ (see $\S 8$ ).

Proposition 6.3. Assume the hypotheses (H1)-(H3); then for any $0<h<1$ and any $x \in \mathbb{R}$ we have

$$
u(h, x)=\mathbb{E} u_{0}\left(Z_{h}(x)\right)+h f\left(\mathbb{E} u_{0}\left(Z_{h}(x)\right)\right)+R_{h}(x)
$$

with the following estimate:

$$
\begin{aligned}
\left|R_{h}(x)\right| \leq & C \mathbb{E}\left\{u _ { 0 } ( Z _ { h } ( x ) ) \left\{\left[\int_{0}^{h} \frac{f \circ u\left(h-s, Z_{s}(x)\right)}{u\left(h-s, Z_{s}(x)\right)} d s\right]^{2}\right.\right. \\
& \left.\left.+\int_{0}^{h} \int_{0}^{s} \frac{f \circ u\left(\theta, Z_{h-\theta}(x)\right)}{u\left(\theta, Z_{h-\theta}(x)\right)} d \theta d s\right\}\right\} \\
& +C \int_{0}^{h} \int_{\mathbb{R}} \mathbb{E}\left[u_{0}\left(Z_{s}(y)\right)-\mathbb{E} u_{0}\left(Z_{s}(y)\right)\right]^{2} P_{h-s}(x, d y) d s \\
& +C h \mathbb{E}\left[u_{0}\left(Z_{h}(x)\right)-\mathbb{E} u_{0}\left(Z_{h}(x)\right)\right]^{2} .
\end{aligned}
$$

For the proof of this proposition we need the following lemma.

Lemma 6.4. There holds

$$
\int_{\mathbf{R}} f\left(\mathbb{E} u_{0}\left(Z_{s}(y)\right)\right) P_{h-s}(x, d y)=f\left(\mathbb{E} u_{0}\left(Z_{h}(x)\right)\right)+R
$$

with

$$
\begin{aligned}
|R| \leq & C \int_{\mathbb{R}} \mathbb{E}\left[u_{0}\left(Z_{s}(y)\right)-\mathbb{E} u_{0}\left(Z_{s}(y)\right)\right]^{2} P_{h-s}(x, d y) \\
& +C \mathbb{E}\left[u_{0}\left(Z_{h}(x)\right)-\mathbb{E} u_{0}\left(Z_{h}(x)\right)\right]^{2} .
\end{aligned}
$$

Proof of Lemma 6.4. Using Lemma 2.7, we have

with

$$
\int_{\mathbf{R}} f\left(\mathbb{E} u_{0}\left(Z_{s}(y)\right)\right) P_{h-s}(x, d y)=\int_{\mathbf{R}} \mathbb{E} f \circ u_{0}\left(Z_{s}(y)\right) P_{h-s}(x, d y)+R_{1}
$$

$$
\left|R_{1}\right| \leq C \int_{\mathbf{R}} \mathbb{E}\left[u_{0}\left(Z_{s}(y)\right)-\mathbb{E} u_{0}\left(Z_{s}(y)\right)\right]^{2} P_{h-s}(x, d y) .
$$

We now note that

$$
\int_{\mathbf{R}} \mathbb{E} f \circ u_{0}\left(Z_{s}(y)\right) P_{h-s}(x, d y)=\mathbb{E} f \circ u_{0}\left(Z_{h}(x)\right),
$$

from which, by applying once again Lemma 2.7 , we get the conclusion.

Proof of Proposition 6.3. Hypothesis (H1) implies that $|f(y) / y| \leq C$ for a suitable $C$ and $0<y \leq 1$; moreover, $f(y) / y$ is continuous in 0 . 
By the Feynman-Kac formula, we have

$$
\begin{aligned}
u(h, x)= & \mathbb{E}\left[u_{0}\left(Z_{h}(x)\right) \exp \left\{\int_{0}^{h} \frac{f \circ u\left(h-s, Z_{s}(x)\right)}{u\left(h-s, Z_{s}(x)\right)} d s\right\}\right] \\
= & \mathbb{E} u_{0}\left(Z_{h}(x)\right)+\mathbb{E} u_{0}\left(Z_{h}(x)\right) \\
& \cdot\left\{\exp \left\{\int_{0}^{h} \frac{f \circ u\left(h-s, Z_{s}(x)\right)}{u\left(h-s, Z_{s}(x)\right)} d s\right\}-1\right\} \\
=: & \mathbb{E} u_{0}\left(Z_{h}(x)\right)+A_{h} .
\end{aligned}
$$

By applying the Taylor formula, we can write

$$
\begin{aligned}
A_{h}= & \mathbb{E} u_{0}\left(Z_{h}(x)\right) \int_{0}^{h} \frac{f \circ u\left(h-s, Z_{s}(x)\right)}{u\left(h-s, Z_{s}(x)\right)} d s \\
& +\frac{1}{2} \mathbb{E} u_{0}\left(Z_{h}(x)\right)\left[\int_{0}^{h} \frac{f \circ u\left(h-s, Z_{s}(x)\right)}{u\left(h-s, Z_{s}(x)\right)} d s\right]^{2} \\
& \cdot \exp \left(\theta_{h} \int_{0}^{h} \frac{f \circ u\left(h-s, Z_{s}(x)\right)}{u\left(h-s, Z_{s}(x)\right)} d s\right)
\end{aligned}
$$

from which we have $A_{h}=B_{h}+R_{h}^{0}$ with

$$
\left|R_{h}^{0}\right| \leq C \mathbb{E} u_{0}\left(Z_{h}(x)\right)\left[\int_{0}^{h} \frac{f \circ u\left(h-s, Z_{s}(x)\right)}{u\left(h-s, Z_{s}(x)\right)} d s\right]^{2}
$$

and

$$
\begin{aligned}
B_{h} & =\mathbb{E} u_{0}\left(Z_{h}(x)\right) \int_{0}^{h} \frac{f \circ u\left(h-s, Z_{s}(x)\right)}{u\left(h-s, Z_{s}(x)\right)} d s \\
& =\mathbb{E} \int_{0}^{h} u_{0}\left(Z_{h}(x)\right) \frac{f \circ u\left(s, Z_{h-s}(x)\right)}{u\left(s, Z_{h-s}(x)\right)} d s \\
& =\int_{0}^{h} \int_{\mathbf{R}} \mathbb{E} u_{0}\left(Z_{s}(y)\right) \frac{f \circ u(s, y)}{u(s, y)} P_{h-s}(x, d y) d s,
\end{aligned}
$$

where, in the last steps, we used the transition property of $P_{\theta}(x, d y)$.

On the other hand, by the same argument used to obtain (6.6), we have

$$
\mathbb{E} u_{0}\left(Z_{s}(y)\right)=u(s, y)+D(s, y)
$$

with, for some $C>0$ large enough,

$$
|D(s, y)| \leq C \mathbb{E} u_{0}\left(Z_{s}(y)\right) \int_{0}^{s} \frac{f \circ u\left(s-\theta, Z_{\theta}(y)\right)}{u\left(s-\theta, Z_{\theta}(y)\right)} d \theta .
$$

Hence, we can write

$$
B_{h}=\int_{0}^{h} \int_{\mathbf{R}} f \circ u(s, y) P_{h-s}(x, d y) d s+E_{h}
$$

with (remembering that $\frac{f(y)}{y}$ is uniformly bounded in $[0,1]$ )

$$
\begin{aligned}
\left|E_{h}\right| & \leq C \int_{0}^{h} \int_{\mathbf{R}} \mathbb{E} u_{0}\left(Z_{s}(y)\right) \int_{0}^{s} \frac{f \circ u\left(s-\theta, Z_{\theta}(y)\right)}{u\left(s-\theta, Z_{\theta}(y)\right)} d \theta P_{h-s}(x, d y) d s \\
& =C \int_{0}^{h} \mathbb{E} u_{0}\left(Z_{h}(x)\right) \int_{0}^{s} \frac{f \circ u\left(s-\theta, Z_{\theta+h-s}(x)\right)}{u\left(s-\theta, Z_{\theta+h-s}(x)\right)} d \theta d s .
\end{aligned}
$$


Finally, by collecting (6.7), (6.8), (6.10), we have

$$
u(h, x)=\mathbb{E} u_{0}\left(Z_{h}(x)\right)+\int_{0}^{h} \int_{\mathbb{R}} f \circ u(s, y) P_{h-s}(x, d y) d s+R_{h}^{1}
$$

with

$$
\begin{array}{rl}
\left|R_{h}^{1}\right| \leq C & \mathbb{E} u_{0}\left(Z_{h}(x)\right) \\
\cdot\left\{\left[\int_{0}^{h} \frac{f \circ u\left(h-s, Z_{s}(x)\right)}{u\left(h-s, Z_{s}(x)\right)} d s\right]^{2}\right. & \left.\quad+\int_{0}^{h} \int_{0}^{s} \frac{f \circ u\left(s-\theta, Z_{\theta+h-s}(x)\right)}{u\left(s-\theta, Z_{\theta+h-s}(x)\right)} d \theta d s\right\} .
\end{array}
$$
that

Therefore, it remains to treat $\int_{0}^{h} \int_{\mathbf{R}} f \circ u(s, y) P_{h-s}(x, d y) d s$. We observe

$$
\begin{aligned}
\int_{0}^{h} & \int_{\mathbf{R}} f \circ u(s, y) P_{h-s}(x, d y) d s \\
& =\int_{0}^{h} \int_{\mathbf{R}} f\left(\mathbb{E} u_{0}\left(Z_{s}(y)\right)-D(s, y)\right) P_{h-s}(x, d y) d s \\
& =\int_{0}^{h} \int_{\mathbb{R}} f\left(\mathbb{E} u_{0}\left(Z_{s}(y)\right)\right) P_{h-s}(x, d y) d s+C_{h}
\end{aligned}
$$

with (using (6.9))

$$
\begin{aligned}
\left|C_{h}\right| & \leq C \int_{0}^{h} \int_{\mathbf{R}}|D(s, y)| P_{h-s}(x, d y) \\
& \leq C \int_{0}^{h} \int_{\mathbf{R}} \mathbb{E} u_{0}\left(Z_{s}(y)\right) \int_{0}^{s} \frac{f \circ u\left(s-\theta, Z_{\theta}(y)\right)}{u\left(s-\theta, Z_{\theta}(y)\right)} d \theta P_{h-s}(x, d y) d s \\
& =C \int_{0}^{h} \mathbb{E} u_{0}\left(Z_{h}(x)\right) \int_{0}^{s} \frac{f \circ u\left(s-\theta, Z_{\theta+h-s}(x)\right)}{u\left(s-\theta, Z_{\theta+h-s}(x)\right)} d \theta d s .
\end{aligned}
$$

We conclude by applying Lemma 6.4 .

Proposition 6.5. (i) Assume that $(\mathrm{H} 1)-(\mathrm{H} 3)$ hold, and that $u_{0}$ belongs to a family of functions satisfying (H5) with weights bounded by $\frac{C}{N}$, the constant $C$ being uniform on the family; then, for any $0<s<h$, we have

$$
\int_{\mathbf{R}} \int_{\mathbf{R}} \mathbb{E}\left[u_{0}\left(Z_{s}(y)\right)-\mathbb{E} u_{0}\left(Z_{s}(y)\right)\right]^{2} P_{h-s}(x, d y) d x \leq C \sqrt{h},
$$

where the constant $C$ depends only on $T$ and the coefficients of the differential operator $L$.

(ii) If $u_{0}$ satisfies $(\mathrm{H} 4)$ instead of the above condition, then we have

$$
\begin{gathered}
\int_{\mathbf{R}} \int_{\mathbf{R}} \mathbb{E}\left[u_{0}\left(Z_{s}(y)\right)-\mathbb{E} u_{0}\left(Z_{s}(y)\right)\right]^{2} P_{h-s}(x, d y) d x \\
\leq C h\left\|\sigma u_{0}^{\prime}\right\|_{L^{2}(\mathbf{R})}+C h^{2}\left\|L u_{0}\right\|_{L^{2}(\mathbf{R})} .
\end{gathered}
$$


Proof. In the case of Hypothesis (H5) we have

$$
\begin{aligned}
\mathbb{E}\left[u_{0}\left(Z_{s}(y)\right)-\mathbb{E} u_{0}\left(Z_{s}(y)\right)\right]^{2} & \\
= & \sum_{i=1}^{N} \mathbb{E}\left\{\omega_{0}^{i} H\left(x_{0}^{i}-Z_{s}(y)\right)-\mathbb{E}\left[\omega_{0}^{i} H\left(x_{0}^{i}-Z_{s}(y)\right)\right]\right\}^{2} \\
& +2 \sum_{i<j} \mathbb{E}\left[\left\{\omega_{0}^{i} H\left(x_{0}^{i}-Z_{s}(y)\right)-\mathbb{E}\left[\omega_{0}^{i} H\left(x_{0}^{i}-Z_{s}(y)\right)\right]\right\}\right. \\
& \left.\cdot\left\{\omega_{0}^{j} H\left(x_{0}^{j}-Z_{s}(y)\right)-\mathbb{E}\left[\omega_{0}^{j} H\left(x_{0}^{j}-Z_{s}(y)\right)\right]\right\}\right] \\
= & : S_{N}(y)+2 T_{N}(y) .
\end{aligned}
$$

Thus, using (3.10) and Corollary 2.1(iii), we have

$$
\begin{aligned}
S_{N}(y) & =\sum_{i=1}^{N}\left(\omega_{0}^{i}\right)^{2}\left(\mathbb{P}\left(x_{0}^{i}>Z_{s}(y)\right)-\left[\mathbb{P}\left(x_{0}^{i}>Z_{s}(y)\right)\right]^{2}\right)^{2} \\
& =\sum_{i=1}^{N}\left(\omega_{0}^{i}\right)^{2} \mathbb{P}\left(x_{0}^{i}>Z_{s}(y)\right) \mathbb{P}\left(x_{0}^{i} \leq Z_{s}(y)\right) \\
& \leq \frac{C}{N^{2}} \sum_{i=1}^{N} \exp \left(-\frac{\left(y-x_{0}^{i}\right)^{2}}{2 \lambda s}\right) .
\end{aligned}
$$

Therefore,

$$
\begin{aligned}
\int_{\mathbf{R}} S_{N}(y) P_{h-s}(x, d y) \\
\quad \leq \frac{C}{N^{2}} \sum_{i=1}^{N} \int_{\mathbf{R}} \exp \left(-\frac{\left(y-x_{0}^{i}\right)^{2}}{2 \lambda s}\right) \frac{1}{\sqrt{h-s}} \exp \left(-\frac{(y-x)^{2}}{2 \lambda(h-s)}\right) d y \\
\leq \frac{C}{N^{2}} \frac{\sqrt{s}}{\sqrt{h}} \sum_{i=1}^{N} \exp \left(-\frac{\left(x-x_{0}^{i}\right)^{2}}{2 \lambda h}\right) \\
\leq \frac{C}{N^{2}} \sum_{i=1}^{N} \exp \left(-\frac{\left(x-x_{0}^{i}\right)^{2}}{2 \lambda h}\right)
\end{aligned}
$$

from which

$$
\int_{\mathbf{R}} \int_{\mathbf{R}} S_{N}(y) P_{h-s}(x, d y) d x \leq \frac{C}{N} \sqrt{h} .
$$

With similar arguments, one can show that

$$
T_{N}(y) \leq C \sqrt{h}
$$

(see the details in the Supplement).

In the case of Hypothesis (H4), we can apply the Itô formula

$$
\begin{aligned}
u_{0}\left(Z_{s}(y)\right)-\mathbb{E} u_{0}\left(Z_{s}(y)\right) & \\
= & \int_{0}^{s}\left[L u_{0}\left(Z_{\theta}(y)\right)-\mathbb{E} L u_{0}\left(Z_{\theta}(y)\right)\right] d \theta \\
& +\int_{0}^{s} \sigma\left(Z_{\theta}(y)\right) u_{0}^{\prime}\left(Z_{\theta}(y)\right) d W_{\theta}
\end{aligned}
$$


hence,

$$
\begin{aligned}
\int_{\mathbb{R}} \mathbb{E} & {\left[u_{0}\left(Z_{s}(y)\right)-\mathbb{E} u_{0}\left(Z_{s}(y)\right)\right]^{2} P_{h-s}(x, d y) } \\
\leq & 2 \int_{\mathbb{R}} \mathbb{E}\left[\int_{0}^{s}\left[L u_{0}\left(Z_{\theta}(y)\right)-\mathbb{E} L u_{0}\left(Z_{\theta}(y)\right)\right] d \theta\right]^{2} P_{h-s}(x, d y) \\
& +2 \int_{\mathbb{R}} \mathbb{E}\left[\int_{0}^{s} \sigma^{2}\left(Z_{\theta}(y)\right) u_{0}^{\prime 2}\left(Z_{\theta}(y)\right) d \theta\right] P_{h-s}(x, d y) \\
= & : 2 A+2 B .
\end{aligned}
$$

We estimate $A$ in the following way:

$$
A \leq \int_{\mathbf{R}} s \int_{0}^{s} \mathbb{E}\left[L u_{0}\left(Z_{\theta}(y)\right)\right]^{2} d \theta P_{h-s}(x, d y)=s \int_{h-s}^{h} \mathbb{E}\left[L u_{0}\left(Z_{\theta}(x)\right)\right]^{2} d \theta .
$$

Using again (2.3), we get

$$
\begin{aligned}
\int_{\mathbb{R}} A d x & \leq C \int_{\mathbb{R}} s \int_{h-s}^{h} \int_{\mathbb{R}}\left[L u_{0}(z)\right]^{2} \frac{1}{\sqrt{\theta}} \exp \left(-\frac{(z-x)^{2}}{2 \lambda \theta}\right) d z d \theta d x \\
& =C s^{2} \int_{\mathbb{R}}\left[L u_{0}(z)\right]^{2} d z .
\end{aligned}
$$

In the same way we have

$$
B \leq C s \int_{\mathbf{R}} \sigma^{2}(z) u_{0}^{\prime 2}(z) d x .
$$

Remark 6.6. From (6.11) and (10.5) (see the Supplement) in the preceding proof, we have also shown that in the case (H5) we have

$$
\int_{\mathbb{R}} \mathbb{E}\left[u_{0}\left(Z_{h}(x)\right)-\mathbb{E} u_{0}\left(Z_{h}(x)\right)\right]^{2} d x \leq C \sqrt{h}
$$

or, more precisely, ${ }^{4}$

$$
\int_{\mathbb{R}} \mathbb{E}\left[u_{0}\left(Z_{h}(x)\right)-\mathbb{E} u_{0}\left(Z_{h}(x)\right)\right]^{2} d x \leq C \sqrt{h}\left(\frac{1}{N}+\frac{C}{N^{2}} \sum_{i<j} \exp \left(-\frac{\left(x_{0}^{i}-x_{0}^{j}\right)^{2}}{8 \lambda h}\right)\right) .
$$

From (6.12), in the case of Hypothesis (H4), we have

$$
\int_{\mathbf{R}} \mathbb{E}\left[u_{0}\left(Z_{h}(x)\right)-\mathbb{E} u_{0}\left(Z_{h}(x)\right)\right]^{2} d x \leq C h .
$$

Proposition 6.7. For $\theta \in[0, h]$, define

$$
\psi_{h, \theta}(x):=\mathbb{E}\left[u_{0}\left(Z_{h}(x)\right) \frac{f \circ u\left(\theta, Z_{h-\theta}(x)\right)}{u\left(\theta, Z_{h-\theta}(x)\right)}\right] .
$$

Then, under (H1)-(H3):

(i) if $u_{0}$ satisfies (H4), there exists a $C>0$ such that for all $h<1$ and for all $\theta \in(0, h)$, we have

$$
\left\|\psi_{h, \theta}(\cdot)\right\|_{L^{1}(\mathbf{R})} \leq C
$$

\footnotetext{
${ }^{4}$ See Remark 6.2.
} 
(ii) if $u_{0}$ belongs to a family of functions satisfying (H5) with weights bounded by $\frac{C}{N}$, the constant $C$ being uniform on the family, there exists a $C>0$ such that for all $h<1$ and for all $\theta \in(0, h)$, we have

$$
\left\|\psi_{h, \theta}(\cdot)\right\|_{L^{1}(\mathbf{R})} \leq C+\frac{C}{N} \sum_{i=1}^{N}\left|x_{0}^{i}\right| .
$$

The result is an easy consequence of Lemma 2.6: see the Supplement.

We remark that

$$
\left[\int_{0}^{h} \frac{f \circ u\left(h-s, Z_{s}(x)\right)}{u\left(h-s, Z_{s}(x)\right)} d s\right]^{2} \leq C h \int_{0}^{h} \frac{f \circ u\left(h-s, Z_{s}(x)\right)}{u\left(h-s, Z_{s}(x)\right)} d s .
$$

Like the preceding proposition, one can show

Proposition 6.8. Define

$$
\psi_{h}(x):=\mathbb{E}\left\{u_{0}\left(Z_{h}(x)\right)\left[\int_{0}^{h} \frac{f \circ u\left(h-s, Z_{s}(x)\right)}{u\left(h-s, Z_{s}(x)\right)} d s\right]^{2}\right\} .
$$

Then, under the hypotheses of Proposition 6.5,

(i) if $u_{0}$ satisfies (H4), there exists a $C>0$ such that for all $h<1$

$$
\left\|\psi_{h}(\cdot)\right\|_{L^{1}(\mathbf{R})} \leq C h^{2}
$$

(ii) if $u_{0}$ belongs to a family of functions satisfying $(\mathrm{H} 5)$ with weights bounded by $\frac{C}{N}$, the constant $C$ being uniform on the family, there exists $a C>0$ such that for all $h<1$

$$
\left\|\psi_{h}(\cdot)\right\|_{L^{1}(\mathbf{R})} \leq C h^{2}\left(1+\frac{1}{N} \sum_{i=1}^{N}\left|x_{0}^{i}\right|\right) .
$$

\section{ESTIMATE OF THE GLOBAL ERROR}

We recall the notation $M=T / h$.

We are now in a position to prove the first part of our main Theorem 4.1.

First, we write $\left\|u(T, \cdot)-\bar{u}_{M}(\cdot)\right\|_{L^{1}(\mathbf{R} \times \Omega)} \leq\left\|\mathbb{E} \bar{u}_{M}(\cdot)-\bar{u}_{M}(\cdot)\right\|_{L^{1}(\mathbf{R} \times \Omega)}+\left\|u(T, \cdot)-\mathbb{E} \bar{u}_{M}(\cdot)\right\|_{L^{1}(\mathbf{R})} \cdot$

In $\S 7.1$, we will bound the first term of the right-hand side by $\frac{C}{\sqrt{N}}+C \sqrt{h}$; in $\S 7.3$, the second term will be bounded by $\frac{C}{N}+C \sqrt{h}$, so that the announced convergence rate (for general functions $b$ and $\sigma$ ) will be established.

7.1. Estimate of $\left\|\mathbb{E} \bar{u}_{M}(\cdot)-\bar{u}_{M}(\cdot)\right\|_{L^{1}(\mathbf{R} \times \Omega)}$. Our objective is to show

Proposition 7.1. There exists a constant $C>0$ such that, for any $h=\frac{T}{M}$, any $p \leq M$, and any $N$,

Proof. Define

$$
\left\|\mathbb{E} \bar{u}_{p}(\cdot)-\bar{u}_{p}(\cdot)\right\|_{L^{1}(\mathbf{R} \times \Omega)} \leq \frac{C}{\sqrt{N}}+C \sqrt{h} .
$$

$$
\hat{u}_{p}(x)=\sum_{i=1}^{N} \omega_{p}^{i} H\left(X_{p h}^{i}-x\right) \text {. }
$$


Using (2.14) and (2.10), we have

$$
\left\|\bar{u}_{p}(\cdot)-\mathbb{E} \bar{u}_{p}(\cdot)-\hat{u}_{p}(\cdot)+\mathbb{E} \hat{u}_{p}(\cdot)\right\|_{L^{1}(\mathbf{R} \times \Omega)}=\mathscr{O}(h) .
$$

Therefore, it is sufficient to prove

Lemma 7.2. There exists a $C>0$ such that, for any $N, h \leq 1$, and $p \leq M=$ $T / h$,

$$
\left\|\hat{u}_{p}(\cdot)-\mathbb{E} \hat{u}_{p}(\cdot)\right\|_{L^{1}(\mathbf{R} \times \Omega)} \leq \frac{C}{\sqrt{N}}+C \sqrt{h} .
$$

Proof of Lemma 7.2. We have (using the fact that the sum of the weights is equal to 1)

$$
\begin{aligned}
\int_{\mathbb{R}} \mid & \sum_{i=1}^{N}\left(\mathbb{E} \omega_{p}^{i} H\left(X_{p h}^{i}-x\right)-\omega_{p}^{i} H\left(X_{p h}^{i}-x\right)\right) \mid d x \\
= & \int_{0}^{+\infty}\left|\sum_{i=1}^{N}\left(\mathbb{E} \omega_{p}^{i} H\left(X_{p h}^{i}-x\right)-\omega_{p}^{i} H\left(X_{p h}^{i}-x\right)\right)\right| d x \\
& \quad+\int_{-\infty}^{0}\left|\sum_{i=1}^{N}\left(\mathbb{E} \omega_{p}^{i} H\left(x-X_{p h}^{i}\right)-\omega_{p}^{i} H\left(x-X_{p h}^{i}\right)\right)\right| d x .
\end{aligned}
$$

We will only consider the first term on the right, the second being treated in the same way. We use the independent weights of $\S 5$. We have

$$
\begin{aligned}
\int_{0}^{+\infty} & \left|\sum_{i=1}^{N}\left(\mathbb{E} \omega_{p}^{i} H\left(X_{p h}^{i}-x\right)-\omega_{p}^{i} H\left(X_{p h}^{i}-x\right)\right)\right| d x \\
\leq & \int_{0}^{+\infty}\left|\sum_{i=1}^{N}\left(\mathbb{E}\left[\rho_{p}^{i} H\left(X_{p h}^{i}-x\right)\right]-\rho_{p}^{i} H\left(X_{p h}^{i}-x\right)\right)\right| d x \\
& +\int_{0}^{+\infty}\left|\sum_{i=1}^{N} \mathbb{E}\left[\left(\omega_{p}^{i}-\rho_{p}^{i}\right) H\left(X_{p h}^{i}-x\right)\right]\right| d x \\
& +\int_{0}^{+\infty} \sum_{i=1}^{N}\left|\omega_{p}^{i}-\rho_{p}^{i}\right| H\left(X_{p h}^{i}-x\right) d x .
\end{aligned}
$$

Using the independence of the $\rho^{i}$ 's and of the $\left(X^{i}\right)$ 's, and bounding the variance by the second moment, one gets

$$
\begin{aligned}
\mathbb{E} \int_{0}^{+\infty} & \left|\sum_{i=1}^{N}\left(\mathbb{E} \omega_{p}^{i} H\left(X_{p h}^{i}-x\right)-\omega_{p}^{i} H\left(X_{p h}^{i}-x\right)\right)\right| d x \\
& \leq \int_{0}^{+\infty} \sqrt{\sum_{i=1}^{N} \mathbb{E}\left(\rho_{p}^{i}\right)^{2} H\left(X_{p h}^{i}-x\right)} d x+2 \sum_{i=1}^{N} \mathbb{E}\left|\omega_{p}^{i}-\rho_{p}^{i}\right|\left|X_{p h}^{i}\right| \\
& \leq \int_{0}^{+\infty} \frac{C}{N} \sqrt{\sum_{i=1}^{N} \mathbb{P}\left(X_{p h}^{i}>x\right)} d x+2 \sqrt{\alpha_{p}} \sum_{i=1}^{N} \sqrt{\mathbb{E}\left|X_{p h}^{i}\right|^{2}}
\end{aligned}
$$


Now we observe that the first term of the right-hand side can be bounded from above by

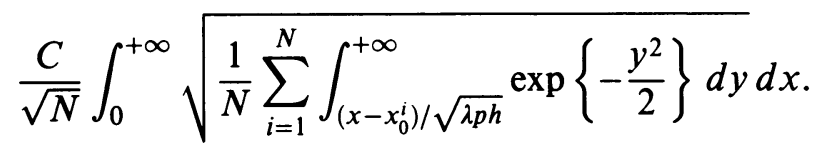

For $x \in[0,+\infty)$ the function

$$
s \stackrel{\mu_{x}}{\stackrel{1}{\sqrt{2 \pi}}} \int_{\left(x-u_{0}^{-1}(s)\right) / \sqrt{\lambda p h}}^{+\infty} \exp \left\{-\frac{y^{2}}{2}\right\} d y
$$

is decreasing from $(0,1)$ to $(0,1)$; therefore, the definition of the $x_{0}^{i}$ implies

$$
\begin{aligned}
\frac{1}{2 N} & \sum_{i=1}^{N} \int_{\left(x-x_{0}^{i}\right) / \sqrt{\lambda p h}}^{+\infty} \exp \left\{-\frac{y^{2}}{2}\right\} d y \\
& \leq \int_{0}^{1} \int_{\left(x-u_{0}^{-1}(s)\right) / \sqrt{\lambda p h}}^{+\infty} \exp \left\{-\frac{y^{2}}{2}\right\} d y d s \\
& =-\int_{\mathbf{R}} \int_{(x-z) / \sqrt{\lambda p h}}^{+\infty} \exp \left\{-\frac{y^{2}}{2}\right\} d y u_{0}^{\prime}(z) d z \\
& \leq-\int_{-\infty}^{x} u_{0}^{\prime}(z) \int_{(x-z) / \sqrt{\lambda p h}}^{+\infty} \exp \left\{-\frac{y^{2}}{2}\right\} d y d z-C \int_{x}^{+\infty} u_{0}^{\prime}(z) d z \\
& \leq-\int_{-\infty}^{+\infty} u_{0}^{\prime}(z) \exp \left\{-\frac{(x-z)^{2}}{2 \lambda p h}\right\} d z+C u_{0}(x) .
\end{aligned}
$$

Using (H4), we deduce for suitable $\lambda_{0}>0$

$$
\frac{1}{N} \sum_{i=1}^{N} \int_{\left(x-x_{0}^{i}\right) / \sqrt{\lambda p h}}^{+\infty} \exp \left\{-\frac{y^{2}}{2}\right\} d y \leq C \exp \left(-\frac{x^{2}}{2 \lambda_{0}(1+p h)}\right)+C u_{0}(x),
$$

so that, by (2.12),

$$
\frac{1}{N} \int_{0}^{+\infty} \sqrt{\sum_{i=1}^{N} \mathbb{P}\left(X_{p h}^{i}>x\right)} d x \leq \frac{C}{\sqrt{N}}
$$

Now, by (5.6),

$$
\sqrt{\alpha_{p}} \sum_{i=1}^{N} \sqrt{\mathbb{E}\left|X_{p h}^{i}\right|^{2}} \leq\left(\frac{C \sqrt{h}}{N}+\frac{C}{N \sqrt{N}}\right) \sum_{i=1}^{N}\left(1+\mathbb{E}\left|X_{p h}^{i}\right|^{2}\right) .
$$

But (see (2.8))

$$
\frac{1}{N} \sum_{i=1}^{N} \mathbb{E}\left|X_{p h}^{i}\right|^{2} \leq \frac{C}{N} \sum_{i=1}^{N}\left(1+\left|x_{0}^{i}\right|^{2}\right) \leq C+\frac{1}{N} \sum_{i=1}^{N}\left|x_{0}^{i}\right|^{2} .
$$

Then we apply (3.13).

7.2. A corollary. As a corollary to the previous subsection, we have the following result: 


\section{Lemma 7.3. Define}

$$
A_{p}^{h}(N):=\int_{\mathbf{R}} \mathbb{E}\left[\int_{\mathbf{R}}\left(\bar{u}_{p}(y)-\mathbb{E} \bar{u}_{p}(y)\right) p_{h}(x, y) d y\right]^{2} d x .
$$

There exists a $C>0$ such that

$$
A_{p}^{h}(N) \leq \frac{C}{\sqrt{N}}+C \sqrt{h}
$$

for any $N, h<1$, and $p \leq M=T / h$.

Proof. First we observe that

$$
A_{p}^{h}(N) \leq \int_{\mathbf{R}} \int_{\mathbf{R}} \mathbb{E}\left[\bar{u}_{p}(y)-\mathbb{E} \bar{u}_{p}(y)\right]^{2} p_{h}(x, y) d y d x
$$

and by Lemma 2.2

$$
A_{p}^{h}(N) \leq(1+C h) \int_{\mathbf{R}} \mathbb{E}\left[\bar{u}_{p}(y)-\mathbb{E} \bar{u}_{p}(y)\right]^{2} d y,
$$

from which, by the boundedness of the function $\bar{u}_{p}$,

$$
A_{p}^{h}(N) \leq C \int_{\mathbf{R}} \mathbb{E}\left|\bar{u}_{p}(y)-\mathbb{E} \bar{u}_{p}(y)\right| d y .
$$

We then apply Lemma 7.2.

7.3. Estimate of $\left\|u(T, \cdot)-\mathbb{E} \bar{u}_{M}(\cdot)\right\|_{L^{1}(\mathbf{R})}$. For any $p=1, \ldots, M$, define $\bar{\nu}_{p}(t, x)$ as the solution to

$$
\left\{\begin{array}{l}
\frac{\partial \bar{\nu}_{p}}{\partial t}=L \bar{\nu}_{p}+f \circ \bar{\nu}_{p} \\
\bar{\nu}_{p}(0, x)=\bar{u}_{p-1}(x)
\end{array}\right.
$$

and consider

$$
\begin{aligned}
\beta_{p} & :=\left\|u(p h, \cdot)-\mathbb{E} \bar{u}_{p}(\cdot)\right\|_{L^{1}(\mathbf{R})} \\
& \leq \underbrace{\left\|u(p h, \cdot)-\mathbb{E} \bar{\nu}_{p}(h, \cdot)\right\|_{L^{1}(\mathbf{R})}}_{\gamma_{p}}+\underbrace{\left\|\mathbb{E} \bar{\nu}_{p}(h, \cdot)-\mathbb{E} \bar{u}_{p}(\cdot)\right\|_{L^{1}(\mathbf{R})}}_{\delta_{p}} .
\end{aligned}
$$

We will show (cf. Proposition 7.6 below) that

$$
\forall p, \quad \beta_{p} \leq C\left(\sqrt{h}+\frac{1}{N}\right)
$$

We first treat

$$
\begin{aligned}
\delta_{p}= & \int_{0}^{+\infty}\left|\mathbb{E} \bar{\nu}_{p}(h, x)-\mathbb{E} \bar{u}_{p}(x)\right| d x \\
& +\int_{-\infty}^{0}\left|\mathbb{E}\left(1-\bar{\nu}_{p}(h, x)\right)-\mathbb{E}\left(1-\bar{u}_{p}(x)\right)\right| d x
\end{aligned}
$$

Our objective is to show 
Proposition 7.4. For all $p$, there holds

$$
\delta_{p} \leq C\left(h^{3 / 2}+\frac{h}{N}\right) .
$$

Proof. We will only consider the first term on the right of (7.3).

Consider $\left(B_{\theta}\right)$, a $\left(\mathscr{G}_{\theta}\right)$-Brownian motion (see the beginning of $\S 3.2$ ), and $\left(\eta_{\theta}(y)\right)$, the solution to

$$
\left\{\begin{array}{l}
d \eta_{\theta}=\sigma\left(\eta_{\theta}\right) d\left(B_{p h+\theta}-B_{p h}\right)-\left\{b\left(\eta_{\theta}\right)-\sigma\left(\eta_{\theta}\right) \sigma^{\prime}\left(\eta_{\theta}\right)\right\} d \theta, \\
\eta_{0}=y .
\end{array}\right.
$$

We stress that, for each $\theta \geq 0, \eta_{\theta}(y)$ is independent of $\mathscr{G}_{p h}$.

We will denote by $\bar{\eta}_{h}(y)$ the approximation of $\eta_{h}(y)$ obtained by applying the Milshtein scheme (2.7) to the stochastic differential equation (7.5).

We first note, using (3.12) and the conventions described in (1.2), that

$$
\begin{aligned}
\mathbb{E} \bar{u}_{p+1}(x)= & \mathbb{E} \sum_{i=1}^{N} \omega_{p+1}^{i} H\left(\bar{X}_{p+1}^{i}-x\right) \\
= & \mathbb{E} \sum_{i=1}^{N}\left[\omega_{p}^{i}\left(1+h f^{\prime} \circ \bar{u}_{p}\left(\bar{X}_{p}^{i}\right)\right)+\mathscr{O}(h) \mathcal{O}\left(\frac{1}{N^{2}}\right)\right] H\left(\bar{\eta}_{h}\left(\bar{X}_{p}^{i}\right)-x\right) \\
= & \mathbb{E} \sum_{i=1}^{N} \omega_{p}^{i} H\left(\bar{\eta}_{h}\left(\bar{X}_{p}^{i}\right)-x\right)+h \mathbb{E} \sum_{i=1}^{N} \omega_{p}^{i} f^{\prime} \circ \bar{u}_{p}\left(\bar{X}_{p}^{i}\right) H\left(\bar{X}_{p}^{i}-x\right) \\
& +\mathbb{E} \mathscr{O}(h) \mathscr{O}\left(\frac{1}{N^{2}}\right) \sum_{i=1}^{N} H\left(\bar{\eta}_{h}\left(\bar{X}_{p}^{i}\right)-x\right) \\
& +h \mathbb{E} \sum_{i=1}^{N} \omega_{p}^{i} f^{\prime} \circ \bar{u}_{p}\left(\bar{X}_{p}^{i}\right)\left(H\left(\bar{\eta}_{h}\left(\bar{X}_{p}^{i}\right)-x\right)-H\left(\bar{X}_{p}^{i}-x\right)\right) .
\end{aligned}
$$

Therefore,

$$
\begin{aligned}
\mathbb{E} \bar{u}_{p+1}(x)= & \mathbb{E} \sum_{i=1}^{N} \omega_{p}^{i} H\left(\bar{\eta}_{h}\left(\bar{X}_{p}^{i}\right)-x\right) \\
& +h \mathbb{E} \sum_{i=1}^{N} \omega_{p}^{i} f^{\prime} \circ \bar{u}_{p}\left(\bar{X}_{p}^{i}\right) H\left(\bar{X}_{p}^{i}-x\right)+\bar{R}(h, p, x)
\end{aligned}
$$

with (we apply (2.4) and (2.8))

$$
\begin{aligned}
\|\bar{R}(h, p, \cdot)\|_{L^{1}\left(\mathbf{R}_{+}\right)} & \leq C h \sqrt{h}+C \frac{h}{N^{2}} \sum_{i=1}^{N} \mathbb{E}\left|\bar{\eta}_{h}\left(\bar{X}_{p}^{i}\right)\right| \\
& \leq C h \sqrt{h}+C \frac{h}{N^{2}} \sum_{i=1}^{N} \mathbb{E}\left|\bar{X}_{p}^{i}\right| \\
& \leq C h \sqrt{h}+C \frac{h}{N^{2}} \sum_{i=1}^{N}\left(1+\left|x_{0}^{i}\right|\right) .
\end{aligned}
$$


Applying (3.13), we deduce ${ }^{5}$

$$
\|\bar{R}(h, p, \cdot)\|_{L^{1}\left(\mathbf{R}_{+}\right)} \leq C h \sqrt{h}+C \frac{h}{N} .
$$

Now, we perform a similar expansion of $\mathbb{E} \bar{\nu}_{p+1}(h, x)$.

Using the representation (3.5), we can also write

$$
\mathbb{E} \bar{\nu}_{p+1}(h, x)=\mathbb{E} \sum_{i=1}^{N} \omega_{p}^{i} \psi\left(\bar{X}_{p}^{i}, x\right),
$$

where

$$
\psi(y, x)=\mathbb{E} H\left(\eta_{h}(y)-x\right) \exp \left(\int_{0}^{h} f^{\prime} \circ \bar{\nu}_{p+1}\left(s, \eta_{s}(y)\right) d s\right) .
$$

Using again (2.14) and the estimate (2.9), one can check that

$$
\psi(y, x)=\mathbb{E} H\left(\bar{\eta}_{h}(y)-x\right) \exp \left(\int_{0}^{h} f^{\prime} \circ \bar{\nu}_{p+1}\left(s, \eta_{s}(y)\right) d s\right)+\psi_{0}(h, y, x)
$$

with

$$
\exists C>0, \quad \forall y \in \mathbb{R}, \quad\left\|\psi_{0}(h, y, \cdot)\right\|_{L^{1}(\mathbf{R})} \leq C h^{3 / 2} .
$$

Therefore,

$$
\begin{aligned}
\mathbb{E} \bar{\nu}_{p+1}(h, x)= & \mathbb{E} \sum_{i=1}^{N} \omega_{p}^{i} H\left(\bar{\eta}_{h}\left(\bar{X}_{p}^{i}\right)-x\right) \\
& +\mathbb{E} \sum_{i=1}^{N} \omega_{p}^{i} \int_{0}^{h} f^{\prime} \circ \bar{\nu}_{p+1}\left(s, \eta_{s}\left(\bar{X}_{p}^{i}\right)\right) d s H\left(\bar{X}_{p}^{i}-x\right) \\
& +\widetilde{R}(h, p, x)
\end{aligned}
$$

with

$$
\|\widetilde{R}(h, p, \cdot)\|_{L^{1}\left(\mathbf{R}_{+}\right)} \leq C h \sqrt{h} .
$$

Therefore, combining (7.11) and (7.8), in view of (7.12) and (7.9), we see that it remains to treat

$$
\phi(h, x):=\mathbb{E} \sum_{i=1}^{N} \omega_{p}^{i}\left(h f^{\prime} \circ \bar{u}_{p}\left(\bar{X}_{p}^{i}\right)-\int_{0}^{h} f^{\prime} \circ \bar{\nu}_{p+1}\left(s, \eta_{s}\left(\bar{X}_{p}^{i}\right)\right) d s\right) H\left(\bar{X}_{p}^{i}-x\right)
$$

and to show that its norm in $L^{1}\left(\mathbb{R}_{+}\right)$can be bounded by $C h \sqrt{h}+\frac{C h}{N}$.

${ }^{5}$ When $f \equiv 0$, this term is absent; this permits us to justify a remark we made in $\S 4$. 
But

$\|\phi(h, \cdot)\|_{L^{1}\left(\mathbf{R}_{+}\right)} \leq \mathbb{E} \sum_{i=1}^{N} \omega_{p}^{i}\left|\bar{X}_{p}^{i}\right|\left|\mathbb{E}^{\mathscr{E}_{p h}}\left[h f^{\prime} \circ \bar{u}_{p}\left(\bar{X}_{p}^{i}\right)-\int_{0}^{h} f^{\prime} \circ \bar{\nu}_{p+1}\left(s, \eta_{s}\left(\bar{X}_{p}^{i}\right)\right) d s\right]\right|$.

Furthermore,

$$
\mathbb{E}^{\mathscr{G}_{p h}} f^{\prime} \circ \bar{\nu}_{p+1}\left(s, \eta_{s}\left(\bar{X}_{p}^{i}\right)\right)=f^{\prime}\left(\mathbb{E}^{\mathscr{G}_{p h}} \bar{\nu}_{p+1}\left(s, \eta_{s}\left(\bar{X}_{p}^{i}\right)\right)\right)+r_{1}(i, p, h, s)
$$

with

$$
\left|r_{1}(i, p, h, s)\right| \leq C \mathbb{E}^{\mathscr{S}_{p h}}\left|\bar{\nu}_{p+1}\left(s, \eta_{s}\left(\bar{X}_{p}^{i}\right)\right)-\mathbb{E}^{\mathscr{G}_{p h}} \bar{\nu}_{p+1}\left(s, \eta_{s}\left(\bar{X}_{p}^{i}\right)\right)\right|^{2}
$$

We now expand $f^{\prime}\left(\mathbb{E}^{\mathscr{G}_{p h}} \bar{\nu}_{p+1}\left(s, \eta_{s}\left(\bar{X}_{p}^{i}\right)\right)\right)$.

Let $\left(\eta_{s}^{i}\right)$ be $N$ independent copies of the process $\left(\eta_{s}\right)$. The representation (3.5) permits us to write

$$
\begin{aligned}
f^{\prime}\left(\mathbb{E}^{\mathscr{G}_{p h}} \bar{\nu}_{p+1}\left(s, \eta_{s}\left(\bar{X}_{p}^{i}\right)\right)\right)= & f^{\prime}\left(\mathbb{E}^{\mathscr{G}_{p h}} \sum_{k=1}^{N} \omega_{p}^{k} H\left(\eta_{s}^{k}\left(\bar{X}_{p}^{k}\right)-\eta_{s}^{i}\left(\bar{X}_{p}^{i}\right)\right)\right) \\
& +\mathscr{O}(h)+\mathscr{O}\left(\frac{1}{N}\right) .
\end{aligned}
$$

Then, if we define

$$
\begin{aligned}
r_{2}(i, p, h, s):= & f^{\prime}\left(\mathbb{E}^{\mathscr{G} p h} \sum_{k=1}^{N} \omega_{p}^{k} H\left(\eta_{s}^{k}\left(\bar{X}_{p}^{k}\right)-\eta_{s}^{i}\left(\bar{X}_{p}^{i}\right)\right)\right) \\
& -f^{\prime}\left(\mathbb{E} \sum_{k=1}^{N} \omega_{p}^{k} H\left(\bar{X}_{p}^{k}-\bar{X}_{p}^{i}\right)\right),
\end{aligned}
$$

we get

$$
\begin{aligned}
f^{\prime}\left(\mathbb{E}^{\mathscr{G} p h} \bar{\nu}_{p+1}\left(s, \eta_{s}\left(\bar{X}_{p}^{i}\right)\right)\right) \\
\quad=f^{\prime}\left(\sum_{k=1}^{N} \omega_{p}^{k} H\left(\bar{X}_{p}^{k}-\bar{X}_{p}^{i}\right)\right)+\mathscr{O}(h)+r_{2}(i, p, h, s)+\mathscr{O}\left(\frac{1}{N}\right) \\
\quad=f^{\prime} \circ \bar{u}_{p}\left(\bar{X}_{p}^{i}\right)+\mathscr{O}(h)+r_{2}(i, p, h, s)+\mathscr{O}\left(\frac{1}{N}\right) .
\end{aligned}
$$

Thus, we have obtained

$$
\begin{aligned}
\|\phi(h, \cdot)\|_{L^{1}\left(\mathbf{R}_{+}\right)} \leq \mathbb{E} \sum_{i=1}^{N} \omega_{p}^{i}\left|\bar{X}_{p}^{i}\right|\left(C h^{2}+\right. & \int_{0}^{h}\left|r_{1}(i, p, h, \theta)\right| d \theta \\
& \left.+\int_{0}^{h}\left|r_{2}(i, p, h, \theta)\right| d \theta+C \frac{h}{N}\right) .
\end{aligned}
$$


As above (see (3.14)), we deduce

$\|\phi(h, \cdot)\|_{L^{1}\left(\mathbf{R}_{+}\right)}$

$$
\begin{aligned}
& \leq C h^{2}+C \mathbb{E} \sum_{i=1}^{N} \omega_{p}^{i}\left|\bar{X}_{p}^{i}\right|\left(\int_{0}^{h}\left|r_{2}(i, p, h, \theta)\right| d \theta\right. \\
& \left.+\int_{0}^{h} \mathbb{E}^{\mathscr{E}_{p h}}\left|\bar{\nu}_{p+1}\left(s, \eta_{s}\left(\bar{X}_{p}^{i}\right)\right)-\mathbb{E}^{\mathscr{G}_{p h}} \bar{\nu}_{p+1}\left(s, \eta_{s}\left(\bar{X}_{p}^{i}\right)\right)\right|^{2} d s+C \frac{h}{N}\right) \\
& \leq C h^{2}+C \underbrace{\mathbb{E} \sum_{i=1}^{N} \omega_{p}^{i}\left|\bar{X}_{p}^{i}\right| \int_{0}^{h}\left|r_{2}(i, p, h, \theta)\right| d \theta}_{A}
\end{aligned}
$$

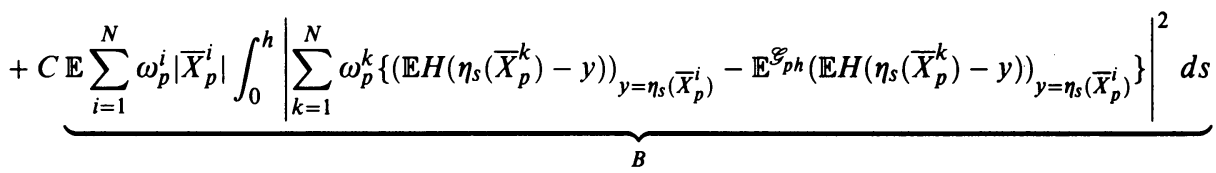

$$
\begin{aligned}
& +C \frac{h}{N^{2}} \sum_{i=1}^{N} \mathbb{E}\left|\bar{X}_{p}^{i}\right|
\end{aligned}
$$

For $A$ we have

$$
\begin{aligned}
A & \leq C \mathbb{E} \sum_{i=1}^{N} \omega_{p}^{i}\left|\bar{X}_{p}^{i}\right| \int_{0}^{h} \mid \mathbb{E}^{\mathscr{G}_{p h}} \sum_{k=1}^{N} \omega_{p}^{k} H\left(\eta_{s}^{k}\left(\bar{X}_{p}^{k}\right)-\eta_{s}^{i}\left(\bar{X}_{p}^{i}\right)\right) \\
& -\mathbb{E} \sum_{k=1}^{N} \omega_{p}^{k} H\left(\bar{X}_{p}^{k}-\bar{X}_{p}^{i}\right) \mid d s \\
& \leq C \mathbb{E} \sum_{i=1}^{N} \omega_{p}^{i}\left|\bar{X}_{p}^{i}\right| \int_{0}^{h} \sum_{k=1}^{N} \omega_{p}^{k} A_{k} d s,
\end{aligned}
$$

where

$$
A_{k}:=\mathbb{E}^{\mathscr{G}_{p h}}\left|H\left(\bar{X}_{p}^{k}-\bar{X}_{p}^{i}\right)-H\left(\eta_{s}^{k}\left(\bar{X}_{p}^{k}\right)-\eta_{s}^{i}\left(\bar{X}_{p}^{i}\right)\right)\right| .
$$

The next steps to prove $A \leq C h \sqrt{h}+\frac{C h}{N}$ are given in the Supplement.

For the term $B$ we observe, using the independence of the particles,

$$
\begin{aligned}
\mathbb{E}^{\mathscr{S}_{p h}}\left|\sum_{k=1}^{N} \omega_{p}^{k}\left\{\left(\mathbb{E} H\left(\eta_{s}\left(\bar{X}_{p}^{k}\right)-y\right)\right)_{y=\eta_{s}\left(\bar{X}_{p}^{i}\right)}-\mathbb{E}^{\mathscr{G}_{p h}}\left(\mathbb{E} H\left(\eta_{s}\left(\bar{X}_{p}^{k}\right)-y\right)\right)_{y=\eta_{s}\left(\bar{X}_{p}^{i}\right)}\right\}\right|^{2} \\
=\mathbb{E}^{\mathscr{S}_{p h}} \sum_{\substack{k=1 \\
k \neq i}}^{N}\left(\omega_{p}^{k}\right)^{2}\left|\left(\mathbb{E} H\left(\eta_{s}\left(\bar{X}_{p}^{k}\right)-y\right)\right)_{y=\eta_{s}\left(\bar{X}_{p}^{k}\right)}-\mathbb{E}^{\mathscr{G}_{p h}}\left(\mathbb{E} H\left(\eta_{s}\left(\bar{X}_{p}^{k}\right)-y\right)\right)_{y=\eta_{s}\left(\bar{X}_{p}^{i}\right)}\right|^{2} \\
+\mathscr{O}\left(\frac{1}{N}\right) \leq \frac{C}{N} .
\end{aligned}
$$

Hence, using (3.14),

$$
B \leq \frac{C h}{N} \mathbb{E} \sum_{i=1}^{N} \omega_{p}^{i}\left|\bar{X}_{p}^{i}\right| \leq \frac{C h}{N} .
$$

We now treat $\gamma_{p}$. 
Proposition 7.5. For all $p$, there holds

$$
\gamma_{p+1} \leq(1+C h) \beta_{p}+C h^{3 / 2}+\frac{C h}{\sqrt{N}} .
$$

Proof. We use the local expansion of $u(p h, \cdot)$ and $\bar{\nu}_{p}(\cdot)$ deduced from Theorem 6.1. Here, Hypothesis (H2) implies that $P_{t}(x, d y)=p_{t}(x, y) d y$. In addition, we apply Proposition 6.1 twice:

- We substitute $u(p h, \cdot)$ to $u_{0}$; then we are in the case where the initial condition satisfies (H4) (from (3.3), (2.3), and (H4), it is easy to obtain the condition on the spatial derivative), and we have

$$
u((p+1) h, x)=\mathbb{E} u\left(p h, Z_{h}(x)\right)+h f\left(\mathbb{E} u\left(p h, Z_{h}(x)\right)\right)+R_{(p+1) h}^{h, 1}(x)
$$

with $\left\|R_{(p+1) h}^{h, 1}(\cdot)\right\|_{L^{1}(\mathbf{R})} \leq C h^{2}$.

- We substitute $\bar{u}_{p}(\cdot)$ to $u_{0}$; then we are in the case where the initial condition satisfies (H5), and we have

$$
\bar{\nu}_{p+1}(x)=\int_{\mathbf{R}} \bar{u}_{p}(y) p_{h}(\cdot, y) d y+h f\left(\int_{\mathbf{R}} \bar{u}_{p}(y) p_{h}(\cdot, y) d y\right)+R_{(p+1) h}^{h, 2}(x)
$$

with

$$
\left\|R_{(p+1) h}^{h, 2}(\cdot)\right\|_{L^{1}(\mathbf{R})} \leq C h \sqrt{h}+C h^{2} \frac{1}{N} \sum_{i=1}^{N}\left|\bar{X}_{p}^{i}\right|,
$$

so that, using (3.14), $\mathbb{E}\left\|R_{(p+1) h}^{h, 2}(\cdot)\right\|_{L^{1}(\mathbb{R})} \leq C h \sqrt{h}$.

Thus, using again (2.13), we get

$$
\begin{aligned}
& \gamma_{p+1}= \| \int_{\mathbf{R}} u(p h, y) p_{h}(\cdot, y) d y+h f\left(\int_{\mathbf{R}} u(p h, y) p_{h}(\cdot, y) d y\right) \\
&-\mathbb{E} \int_{\mathbf{R}} \bar{u}_{p}(y) p_{h}(\cdot, y) d y-h \mathbb{E} f\left(\int_{\mathbf{R}} \bar{u}_{p}(y) p_{h}(\cdot, y) d y\right) \\
&+R_{(p+1) h}^{h, 1}(\cdot)-\mathbb{E} R_{(p+1) h}^{h, 2}(\cdot) \|_{L^{1}(\mathbf{R})} \\
& \leq \quad \int_{\mathbf{R}}\left|u(p h, y)-\mathbb{E} \bar{u}_{p}(y)\right| \int_{\mathbf{R}} p_{h}(x, y) d x d y+C h^{3 / 2} \\
&+C h \int_{\mathbf{R}}\left|u(p h, y)-\mathbb{E} \bar{u}_{p}(y)\right| \int_{\mathbf{R}} p_{h}(x, y) d x d y \\
&+C h \int_{\mathbf{R}} \mathbb{E}\left(\int_{\mathbf{R}} \bar{u}_{p}(y) p_{h}(x, y) d y-\mathbb{E} \int_{\mathbf{R}} \bar{u}_{p}(y) p_{h}(x, y) d y\right)^{2} d x .
\end{aligned}
$$

Applying Lemmas 2.2 and 7.3, one obtains

$$
\gamma_{p+1} \leq(1+C h) \beta_{p}+C h^{3 / 2}+\frac{C h}{\sqrt{N}} .
$$

Finally, we can prove

Proposition 7.6. For all $p$, there holds

$$
\beta_{p} \leq C\left(\sqrt{h}+\frac{1}{\sqrt{N}}\right) .
$$


Proof. We use the definition (7.2) and the estimates (7.14) and (7.4) to get

$$
\beta_{p+1} \leq(1+C h) \beta_{p}+C h^{3 / 2}+\frac{C h}{\sqrt{N}} .
$$

In the proof of Lemma 5.5, we remarked that $\gamma_{1}=\left\|u(h, \cdot)-v^{N}(h, \cdot)\right\|_{L^{1}(\mathbf{R})}$ can be bounded by $C\left\|\mathbb{E}\left|\left(u_{0}-\bar{u}_{0}\right)\left(Z_{h}(\cdot)\right)\right|\right\|_{L^{1}(\mathbf{R})} ;$ as $\bar{u}_{0} \geq u_{0}$ for $x<x_{0}^{N-1}<$ $C(1+\sqrt{\log N})$, using $u(x)=-\int_{x}^{+\infty} u^{\prime}(y) d y,(2.2)$, and (H4), we get, for some $C>0$ large enough,

$$
\begin{aligned}
\gamma_{1} \leq & C \int_{|x|<C+C \sqrt{\log N}}\left(\bar{u}_{0}(x)-u_{0}(x)\right) d x \\
& +C \int_{x \leq-C-C \sqrt{\log N}}\left(1-u_{0}(x)\right) d x+C \int_{x \geq C+C \sqrt{\log N}} u_{0}(x) d x \\
\leq & \frac{C \sqrt{\log N}}{N}+\frac{c}{N} .
\end{aligned}
$$

Thus, $\beta_{1} \leq C\left(\frac{1}{\sqrt{N}}+h^{3 / 2}+\frac{h}{N}\right)$, and we can proceed by induction to end the proof.

\section{The CaSe of CONSTANT COEFFicients}

In this section, we explain what must be changed in the proof to get the better estimate $\frac{C}{\sqrt{N}}+C h$ for the error when the coefficients of $L$ are constant.

Without loss of generality, we can assume that $b \equiv 0$ and $\sigma \equiv 1$. In that case we have that $\bar{X}_{p}^{i}=X_{p}^{i}=x_{0}^{i}+W_{p h}^{i}$.

First, one remarks that the expansion in Remark 5.1 can then be changed to

$$
\begin{aligned}
\omega_{p+1}^{i}-\rho_{p+1}^{i}= & \omega_{p}^{i}-\rho_{p}^{i}+h \omega_{p}^{i}\left\{f^{\prime} \circ \bar{u}_{p}\left(\bar{X}_{p}^{i}\right)-f^{\prime} \circ u\left(p h, \bar{X}_{p}^{i}\right)\right\} \\
& +h\left(\omega_{p}^{i}-\rho_{p}^{i}\right) f^{\prime} \circ u\left(p h, \bar{X}_{p}^{i}\right)+\mathscr{O}\left(\frac{h}{N^{2}}\right),
\end{aligned}
$$

so that the inequality $(5.2)$ can be modified to

$$
\begin{aligned}
\alpha_{p+1}^{i} \leq & (1+C h) \alpha_{p}^{i}+\frac{C h}{N} \sqrt{\alpha_{p}^{i}} \sqrt{\mathbb{E}\left|\bar{u}_{p}\left(\bar{X}_{p}^{i}\right)-u\left(p h, \bar{X}_{p}^{i}\right)\right|^{2}} \\
& +C \frac{h^{2}}{N^{2}} \mathbb{E}\left|\bar{u}_{p}\left(\bar{X}_{p}^{i}\right)-u\left(p h, \bar{X}_{p}^{i}\right)\right|^{2}+C \frac{h}{N^{3}} .
\end{aligned}
$$

One can readily show that $(5.4)$ can be reduced to

$$
\mathbb{E}\left|\bar{u}_{p}\left(\bar{X}_{p}^{i}\right)-u_{p}^{*}\left(\bar{X}_{p}^{i}\right)\right|^{2} \leq N^{2} \alpha_{p} .
$$

Therefore, with the same arguments as in the proof of Proposition 5.8, one can show that the inequality (5.6) can be modified to

$$
\forall p, \quad \alpha_{p} \leq \frac{C h^{2}}{N^{2}}+\frac{C}{N^{3}} .
$$

This remark permits us to change the last lines of the proof of Lemma 7.2, so that one gets

$$
\left\|\hat{u}_{p}(\cdot)-\mathbb{E} \hat{u}_{p}(\cdot)\right\|_{L^{1}(\mathbf{R} \times \Omega)} \leq \frac{C}{\sqrt{N}}+C h .
$$


Consequently, the conclusion of Lemma 7.3 becomes: there exists a constant $C>0$ such that

$$
A_{p}^{h}(N) \leq \frac{C}{\sqrt{N}}+C h
$$

for any $N, h \leq 1$, and $p \leq M=T / h$.

Now, we recall the inequality (6.5). This permits us to modify the beginning of the proof of Proposition 7.5 in the following way:

$$
\begin{aligned}
\gamma_{p+1}= & \| \int_{\mathbb{R}} u(p h, y) p_{h}(\cdot, y) d y+h f\left(\int_{\mathbb{R}} u(p h, y) p_{h}(\cdot, y) d y\right) \\
-\mathbb{E} \int_{\mathbb{R}} \bar{u}_{p}(y) p_{h}(\cdot, y) d y-h \mathbb{E} f\left(\int_{\mathbb{R}} \bar{u}_{p}(y) p_{h}(\cdot, y) d y\right) & +R_{(p+1) h}^{h, 1}(\cdot)+\mathbb{E} R_{(p+1) h}^{h, 2}(\cdot) \|_{L^{1}(\mathbf{R})} \\
\leq & \int_{\mathbb{R}}\left|u(p h, y)-\mathbb{E} \bar{u}_{p}(y)\right| \int_{\mathbb{R}} p_{h}(x, y) d x d y+C h^{2}+C \frac{h \sqrt{h}}{N} \\
& +C h \int_{\mathbb{R}}\left|u(p h, y)-\mathbb{E} \bar{u}_{p}(y)\right| \int_{\mathbb{R}} p_{h}(x, y) d x d y \\
& +C h \int_{\mathbb{R}} \mathbb{E}\left(\int_{\mathbb{R}} \bar{u}_{p}(y) p_{h}(x, y) d y-\mathbb{E} \int_{\mathbb{R}} \bar{u}_{p}(y) p_{h}(x, y) d y\right)^{2} d x .
\end{aligned}
$$

Thus, it remains to check that we can improve the estimate for $\delta_{p}$. Namely, instead of (7.4), we have

$$
\forall p, \delta_{p} \leq C\left(h^{2}+\frac{h}{N}\right) .
$$

Actually, one just has to consider (7.6) and (7.10): now $\bar{\eta}_{h}\left(\bar{X}_{p}^{i}\right)$ and $\eta_{h}\left(\bar{X}_{p}^{i}\right)$ are equal, thus the conclusion is straightforward.

\section{Conclusion}

We have constructed a stochastic particle algorithm for general one-dimensional reaction-diffusion-convection PDEs, by establishing a convenient probabilistic representation of the solution and discretizing it in space and time.

We have given its rate of convergence, which also proves a conjecture of Puckett concerning this method for the KPP equation.

\section{BIBLIOGRAPHY}

1. A. Bensoussan and J. L. Lions, Applications des inéquations variationnelles en contrôle stochastique, Dunod, Paris, 1978.

2. P. Cannarsa and V. Vespri, Generation of analytic semigroups by elliptic operators with unbounded coefficients, SIAM J. Math. Anal. 18 (1987), 857-872.

3. B. Chauvin and A. Rouault, KPP equation and supercritical branching Brownian motion in the subcritical speed area. Application to spatial trees, Probab. Theory Related Fields 80 (1988), 299-314.

4. _ A stochastic simulation for solving scalar reaction-diffusion equations, Adv. Appl. Probab. 12 (1990), 88-100.

5. Supercritical branching Brownian motion and K-P-P equation in the critical speed-area, Math. Nachr. 149 (1990), 41-59. 
6. A. Friedman, Stochastic differential equations and applications, Vol. 1, Academic Press, New York, 1975.

7. T. Hida, Brownian motion, Springer-Verlag, Berlin and New York, 1980.

8. H. Kunita, Stochastic differential equations and stochastic flows of diffeomorphisms, Ecole d'Eté de Saint-Flour XII, Lecture Notes in Math., vol. 1097, Springer-Verlag, Berlin and New York, 1984.

9. G. N. Milshtein, Approximate integration of stochastic differential equations, Theory Probab. Appl. 19 (1974), 557-562.

10. A. Pazy, Semigroups of linear operators and applications to partial differential equations, Springer-Verlag, Berlin and New York, 1983.

11. G. Da Prato and E. Sinestrari, Differential operators with non dense domain, Ann. Scuola Norm. Sup. Pisa Cl. Sci. (4) 14 (1987), 285-344.

12. E. G. Puckett, Convergence of a random particle method to solutions of the Kolmogorov equation, Math. Comp. 52 (1989), 615-645.

13. F. Rothe, Global solutions of reaction-diffusion systems, Lecture Notes in Math., vol. 1072, Springer-Verlag, Berlin and New York, 1984.

14. A. S. Sherman and C. S. Peskin, A Monte Carlo method for scalar reaction diffusion equations, SIAM J. Sci. Statist. Comput. 7 (1986), 1360-1372.

15. B. Stewart, Generation of analytic semigroups by strongly elliptic operators, Trans. Amer. Math. Soc. 199 (1974), 141-162.

16. D. Talay, Simulation and numerical analysis of stochastic differential systems: a review, Rapport de Recherche INRIA, vol. 1313, 1990 (and to appear in Effective Stochastic Analysis (P. Kree and W. Wedig, eds.), Springer-Verlag).

Laboratoire de Mathematiques Appliquées URA-CNRS 1501, Universite Blaise Pascal, 63177 Aubiere Cedex, France

E-mail address: bernardQucfma.univ-bpclermont.fr

INRIA, 2004, Route des Lucioles, Sophia-ANtipolis, B.P. 109, 06561 Valbonne Cedex, FRANCE

E-mail address: talay@sophia.inria.fr

Dipartimento di Matematica, Università di Trento, 38050 Povo (TN), Italy

E-mail address: tubaro@itnvax.science.unitn.it 


\title{
Supplement to
}

RATE OF CONVERGENCE OF A STOCHASTIC PARTICLE METHOD FOR THE KOLMOGOROV EQUATION WITH VARIABLE COEFFICIENTS

\author{
PIERRE BERNARD, DENIS TALAY, AND LUCIANO TUBARO
}

\section{Complementary proofs}

Proof of Lemma 2.2. Let $L$ be the infinitesimal generator of $\left(\xi_{t}\right)$; then the function

$$
q(t, y)=\int_{\boldsymbol{R}} p_{t}(x, y) d x
$$

satisfies the equation (cf. the Problem 10 of Ch.6 in [6])

$$
\left\{\begin{array}{l}
\frac{\partial q}{\partial t}(t, y)=L^{*} q(t, y)=\frac{1}{2} \frac{\partial^{2}}{\partial y^{2}}\left(\sigma_{0}^{2}(y) q(t, y)\right)-\frac{\partial}{\partial y}\left(b_{0}(y) q(t, y)\right) \\
\lim _{t \rightarrow 0} q(t, y)=1
\end{array}\right.
$$

Denote by $\left(\alpha_{t}(y)\right)$ the diffusion issued from $y$ with infinitesimal generator $\left(\sigma_{0}(x) \sigma_{0}^{\prime}(x)-\right.$ $\left.b_{0}(x)\right) \frac{\partial}{\partial x}+\frac{1}{2} \sigma_{0}^{2}(x) \frac{\partial^{2}}{\partial x^{2}}$. We have, denoting $a_{0}(x)=\sigma_{0}^{2}(x)$ (see again the Ch.6 in $[6]$, e.g.)

$$
q(t, y)=\mathbb{E} \exp \left(\int_{0}^{t}\left(\frac{1}{2} a_{0}^{\prime \prime}\left(\alpha_{s}(y)\right)-b_{0}^{\prime}\left(\alpha_{s}(y)\right)\right) d s\right)
$$

Proof of Lemma 2.6. We will only treat the first case; the (H5) case just calls for local and easy modifications.

We introduce the process $\left(Z_{t}(x)\right)$, solution to

$$
d Z_{t}=b\left(Z_{t}\right) d t+\sigma\left(Z_{t}\right) d B_{t}, \quad Z_{0}(x)=x .
$$

Since $\frac{f o u}{u}$ is bounded, the Feynman-Kac formula

$$
u(t, x)=\boldsymbol{E} u_{0}\left(Z_{t}(x)\right) \exp \left(\int_{0}^{t} \frac{f \circ u}{u}\left(s, Z_{s}(x)\right) d s\right)
$$



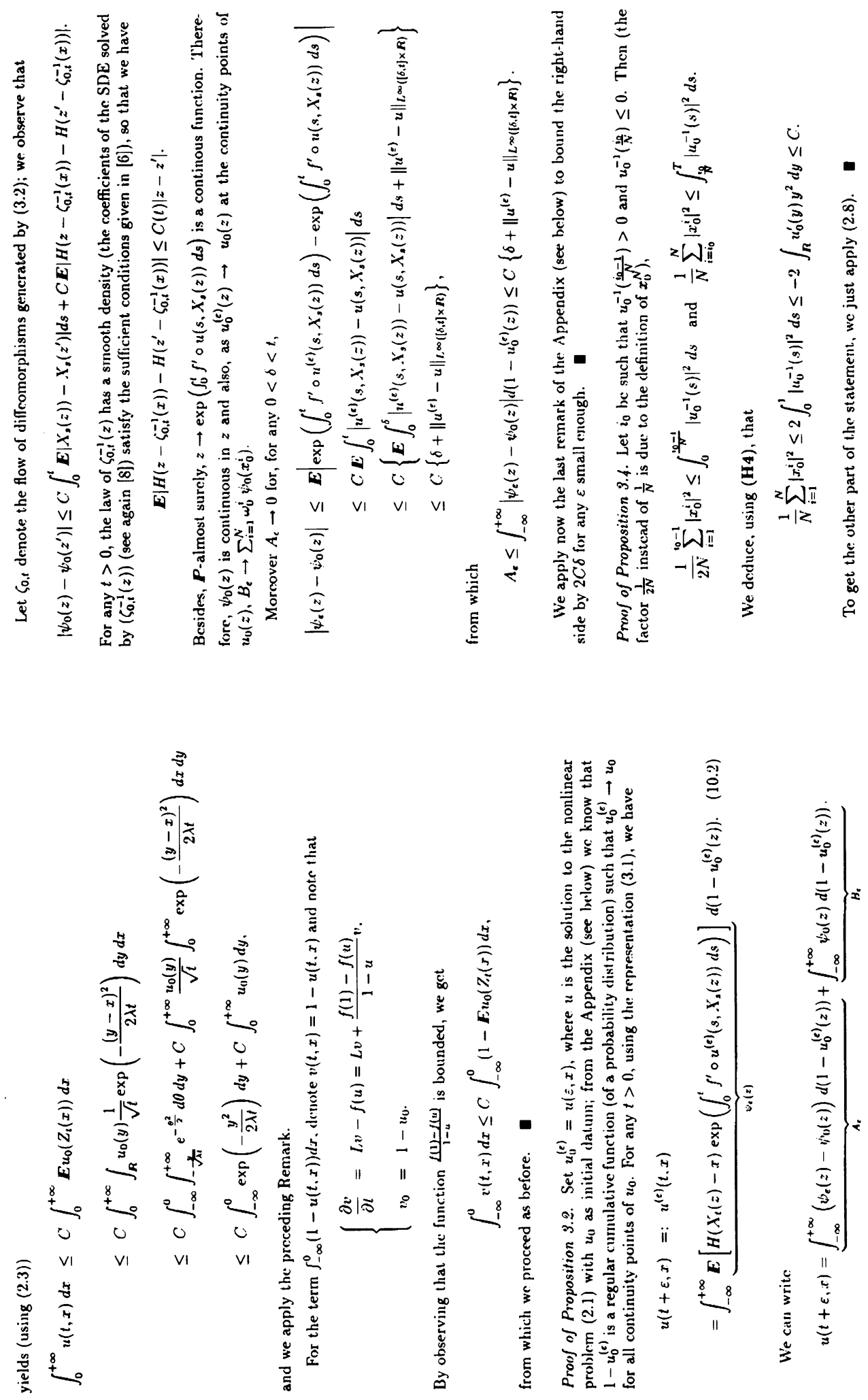

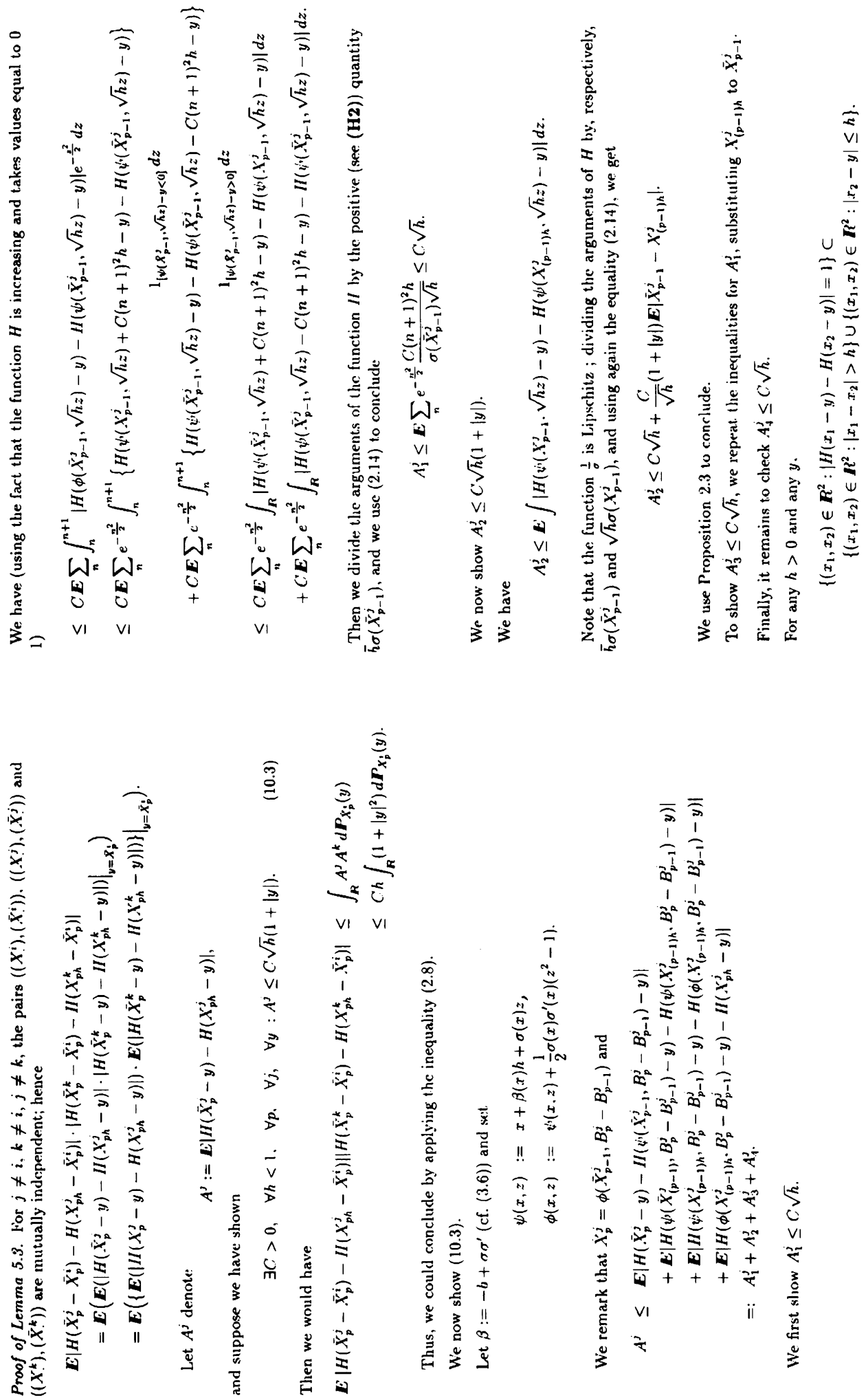

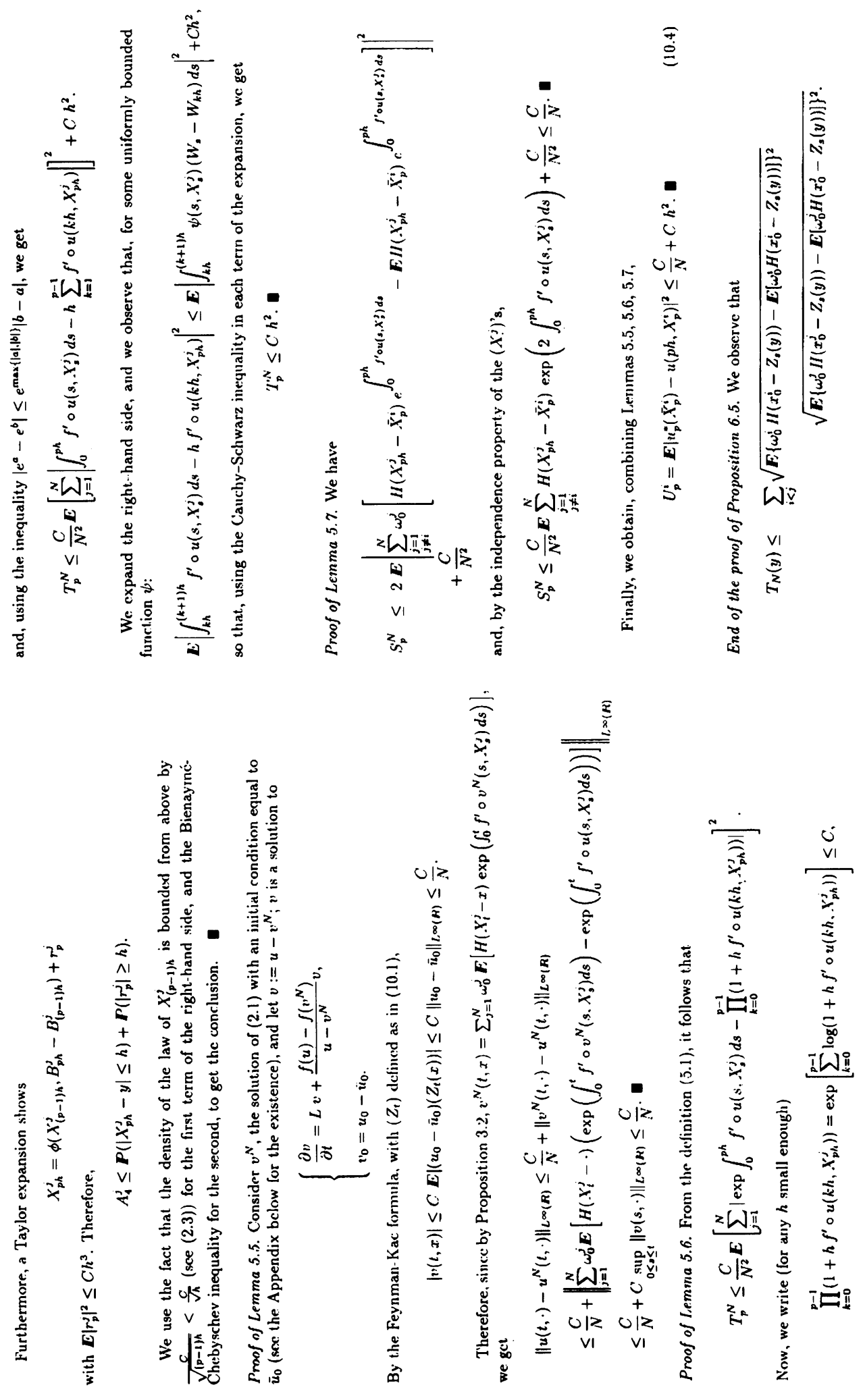

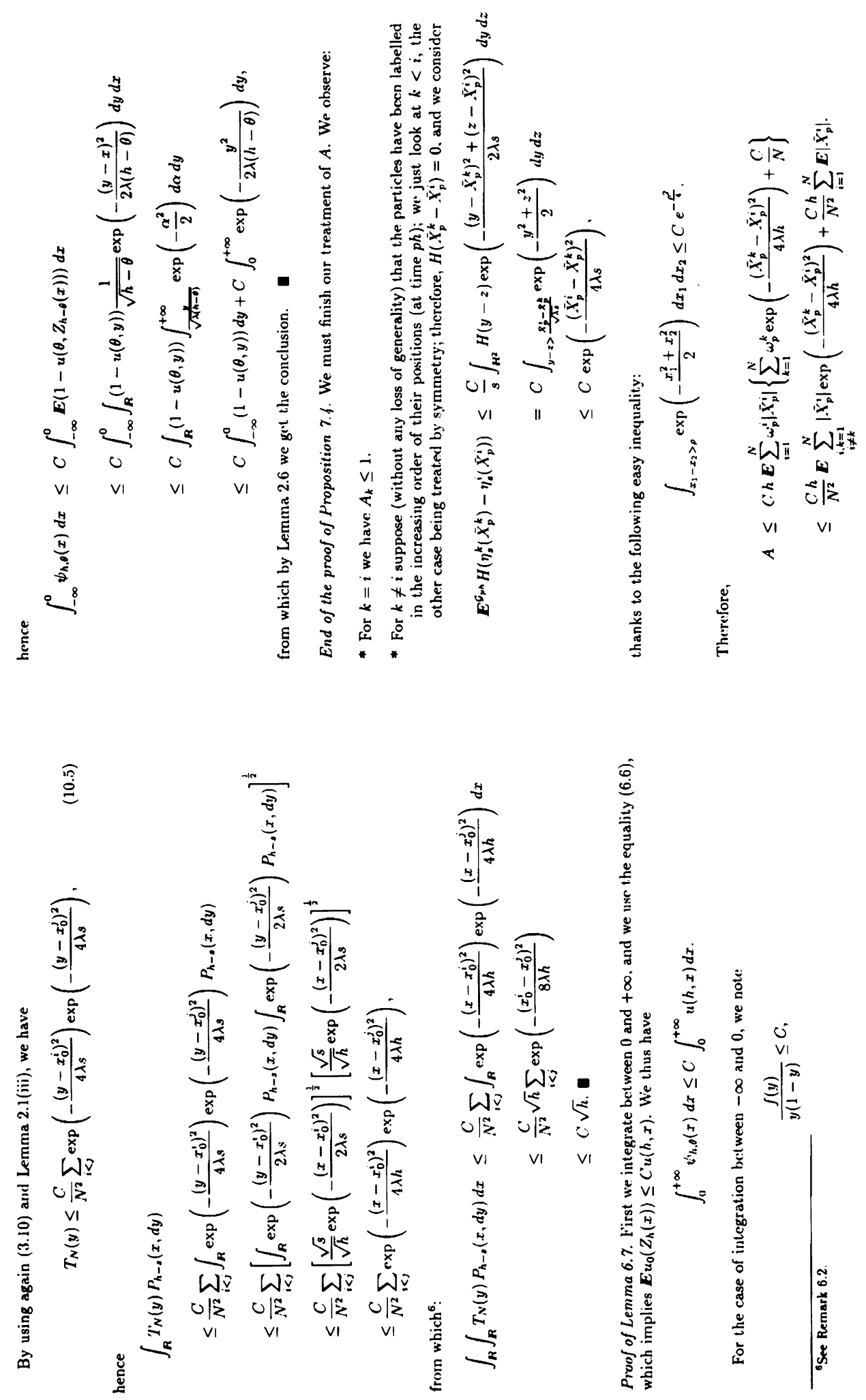

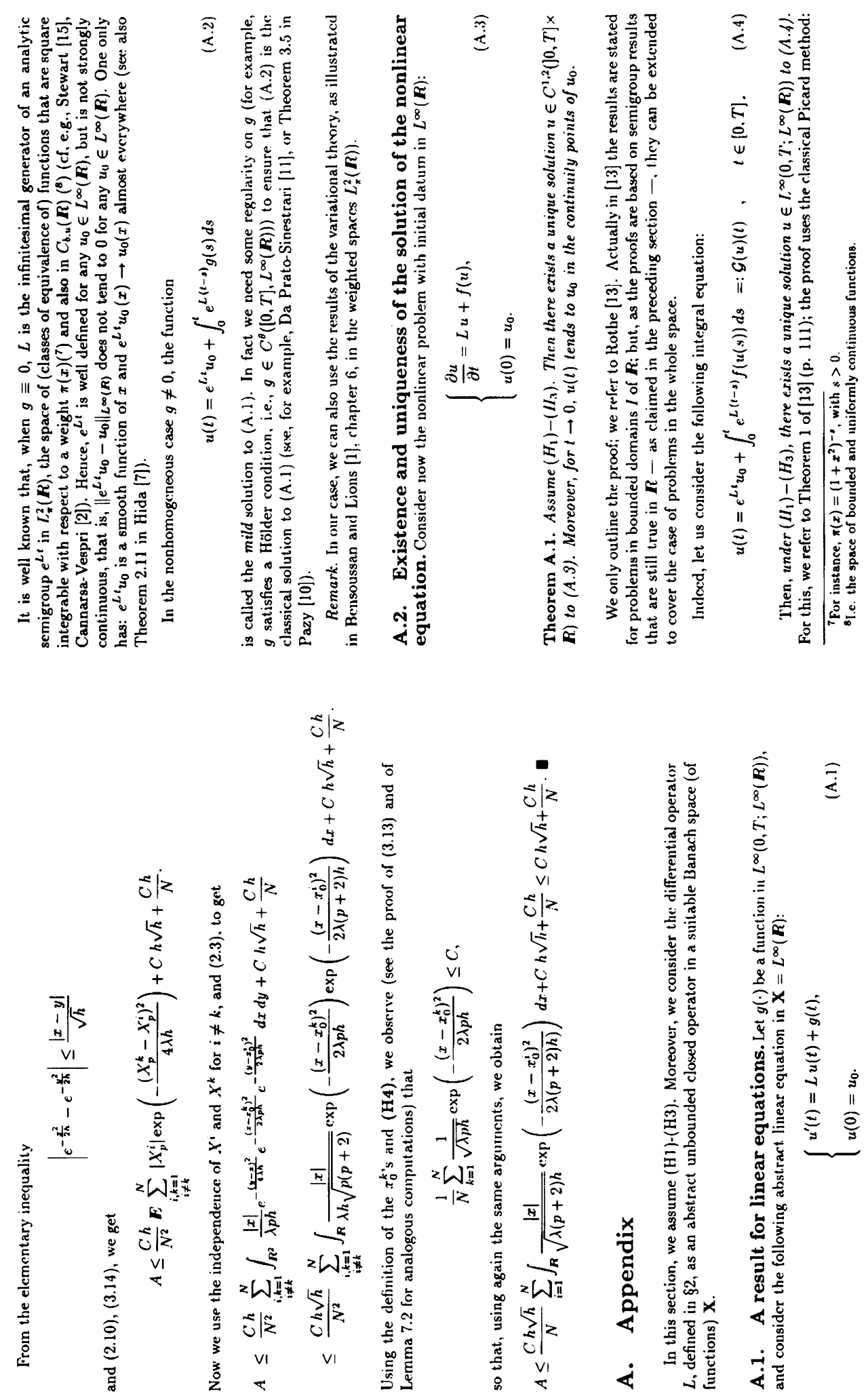


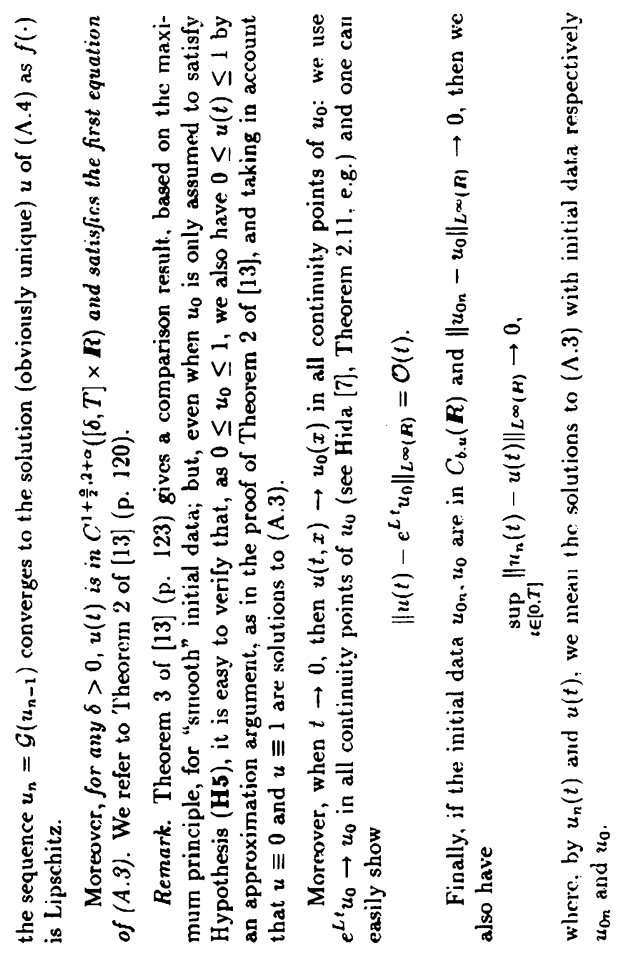

